\title{
Walking of biped with passive exoskeleton: evaluation of energy consumption.
}

\author{
Y. Aoustin ${ }^{1}$ and A. M. Formalskii ${ }^{2}$, \\ 1 1, rue de la Noë, BP 92101. 44321 Nantes, \\ France, \\ Laboratoire des Sciences du Numérique \\ de Nantes, UMR 6004, \\ CNRS, École Centrale de Nantes, Université \\ de Nantes, France \\ Yannick.Aoustin@ls2n.fr. \\ ${ }^{2}$ Institute of Mechanics, Lomonosov Moscow \\ State University, \\ 1, Michurinskii Prospect, Moscow, 119192, \\ Russia, \\ formal@imec.msu.ru
}

the date of receipt and acceptance should be inserted later

\begin{abstract}
The paper aim is to show theoretically the feasibility and efficiency of a passive exoskeleton for human walking and carrying a load. Human is modeled using a planar bipedal anthropomorphic mechanism. This mechanism consists of a trunk and two identical legs; each leg consists of a thigh, shin, and foot (massless). The exoskeleton is considered also as an anthropomorphic mechanism. The shape and the degrees of freedom of the exoskeleton are identical to the biped (to human) - the topology of the exoskeleton is the same as of the biped (human). Each of models of human and of exoskeleton has seven links and six joints. The hip-joint connects the trunk and two thighs of the two legs. If the biped is equipped with an exoskeleton, then the links of this exoskeleton are attached to the corresponding links of the biped and the corresponding hip-, knee-, and ankle-joints coincide. We compare the walking gaits of a biped alone (without exoskeleton) and of a biped equipped with exoskeleton; for both cases the same load is transported. The problem is studied in the framework of ballistic walking model. During the ballistic walking of the biped with exoskeleton the knee of the support leg is locked, but the knee of the swing leg is unlocked. The locking and unlocking can be realized in the knees of the exoskeleton by any mechanical brake devices without energy consumption. There are not any actuators in the exoskeleton. Therefore, we call it passive exoskeleton. The walking of the biped consists of alternating single- and double-support phases. In our study, the double-support phase is assumed as instantaneous. At the instant of this phase, the knee of the previous swing leg is locked and the knee of the previous support leg is unlocked. Numerical results show that during the load transport the human with the exoskeleton spends less energy than human alone. For transportation of a load with mass $40 \mathrm{~kg}$, the economy of the energy is approximately $28 \%$, if the length of the step and its duration are equal to
\end{abstract}


$0.5 \mathrm{~m}$ and $0.5 \mathrm{~s}$ respectively.

Keywords: Human, Bipedal model, Massless feet, Passive Exoskeleton, Ballistic walking, Single-support phase, Instantaneous double-support, Impulsive torque, Optimization, Energy consumption.

\section{Introduction}

Exoskeleton robots are integrated mechanical devices (mechanisms), that can be wearable and attached to the human body. Among them there are external structures that can support a human's body. Research in powered human exoskeleton devices began in the late 1960s for military purposes, [1]. There are currently two families of exoskeleton. Those that are walking assistances and those dedicated to increasing the user's carrying capacity. Among wearable exoskeletons for the walking assistance, that are currently commercially available in the market we can list several examples. $H A L$ (Hybrid Assistive Limb) defines a set of wearable exoskeletons, that are designed for rehabilitation, rescue support and entertainment, [2]. It exists in several versions such as full body, lower body and one leg versions, [3]. The actuation mechanism of $H A L$ is based on surface electromyography signals that provide the system the user's intended movements. Argo Medical Technologies Inc. designs a wearable motorized medical suit $\operatorname{ReWalk}^{T M}$ that are dedicated for therapeutic activities. Its knee- and hip-joints are actuated in the sagittal plane, [4]. Ekso Bionics (earlier Berkeley Bionics) a US company has unveiled a rehabilitation exoskeleton named as EKSO. This company received US FDA (United States Food and Drug Administration) approval to market the exoskeleton for hospital use in 2012 in USA, see [5]. INDEGO exoskeleton is a prototype developed in the University of Vanderbilt at Tennessee, United States, [6]. This lower limb exoskeleton is proposed in to assist persons with paraplegia. For a given assistance device with ballistic motions and impulsive impacts it is theoretically shown in [7] that an assistance at the level of hips only is a good compromise.

Among the second family of exoskeletons let us remark, without being exhaustive, several realizations. HARDIMAN, (Human Augmentation Research and Development Investigation, MANipulation) was an enormous powered exoskeleton (with mass $680 \mathrm{~kg}$ ). It was hydraulically powered. It can drastically increase the strength capabilities of the wearer, (approximately 25:1, actual weight to perceived weight), see [8] and [5]. However it presented balancing problems. Vukobratovic and his associates at the Mihailo Pupin Institute in Belgrade in 1960s and 1970s started with a passive device for measuring the kinematics of walking, see [9]. Then this work progressed to the development of powered pneumatic exoskeletons. The design of the exoskeletons is currently an important area of research in performance enhancement but also in rehabilitation robotics, injury prevention. They can enhance strength and endurance during locomotion of for example military, firemen or life savers with heavy load. The University of California, Berkeley in collaboration with Schilling Robotics, developed the exoskeleton BLEEX (Berkeley Lower Extremity Exoskeleton), see [10], [11], and [12]. A person who is equipped with a $B L E E X$ can carry a rucksack of mass $75 \mathrm{~kg}$ while walking at $0.9 \mathrm{~m} / \mathrm{s}$ and can walk at speeds of up to $1.3 \mathrm{~m} / \mathrm{s}$. The mass of an exoskeleton depends on the actuators. For the second generation of the Berkeley exoskeleton, in part due to the implementation of electric actuation with a hydraulic transmission system, it is approximately $14 \mathrm{~kg}$. Body Extender is a fully actuated body extender 
to amplify the force of a human operator. It has been designed, realized and tested at Percro (Laboratory of Perceptual Robotics), see [13] and [14]. This exoskeleton allows to lift a load that is equal to $100 \mathrm{~kg}$. NAEIES (Naval Aeronautical Engineering Institute Exoskeleton Suit) was developed to walk in different terrains with a heavy load. It is foreseen to be used in mountaineering, firefighting and disaster relief situations. For each leg there are six degrees of freedom $(D O F)$, two revolute joints are at the hip, one revolute joint at the knee and three revolute joints at the ankle, see [15]. The company Raytheon Sarcos, after the robotics suit XOS 1, proposes a second-generation robotics suite XOS 2 for the US Army, [5]. This wearable robotic suit is powered with rotary hydraulic actuators. It uses force sensing to control the motion and provides autonomous power supply to the user. This very powerful exoskeleton is targeted for military purposes to allow the wearer to lift heavy object at a ratio of 17:1 (actual weight to perceived weight).

The relation between human and exoskeleton is also a complex task. Strausser and Kazerooni [16] proposed a Humanoid Machine Interface (HMI) for a mobile medical exoskeleton to allow the users to stand up, walk, and sit down independently. Current trends and major challenges in the development of assistance devices are discussed in [14].

Then the idea to limit the number of actuators is also explored. Through simulations van den Bogert, see [17], proved that an exoskeleton with passive elastic devices can reduce muscle force and metabolic cost of human in walking. As a consequence a quasi-passive exoskeleton concept has been developed at the Massachusetts Institute of Technology Media Laboratory, see [18]. The objective is to exploit the passive dynamics of human walking in order to create lighter and more efficient exoskeleton devices. There are not any actuators for adding power at the joints of the MIT exoskeleton. The quasi-passive elements of the exoskeleton, that are springs and variable dampers, have been chosen from an analysis of the kinetics and kinematics of human walking. Another quasi-passive exoskeleton has been designed at University of Delaware, Newark, see [19] and [20] for the leg of a human subject. This gravity balancing exoskeleton, also does not use any motors, but through springs and a four bars mechanism is able to partially or fully unload the hip- and knee-joints from the gravity throughout the range-of-motion of the leg. Dariush, see [21] proposed a strategy for a partition between natural voluntary control by the central nervous system of human and artificial assist by the exoskeleton controller. In [22] a passive exoskeleton with artificial tendons is proposed. However, authors showed that the effect of the artificial tendons on the energy expenditure while walking is much lower than expected. A realistic $2 \mathrm{D}$ model is defined for the creation of a quasi-passive energy-efficient power-assisted lower-limb exoskeleton in [23]. A novel lower limb exoskeleton is presented in [24] to combine the body weight support function provided to the user and the high portability of crutches and walkers.

Despite all these remarkable contributions, devices are still relatively heavy wearable versus the weight of a human. They are efficient to balance a static payload, but some progresses are necessary to balance the dynamic effects of the payload. It is not yet demonstrated that an exoskeleton is more efficient to reduce the metabolic cost of transport than the load-carriage with a standard rucksack. The location and the number of actuators of an exoskeleton and its autonomy remain a difficult challenge.

The paper aim is to prove theoretically the feasibility and the efficiency of the passive exoskeleton with locked knee of its support leg. The advantages of the passive exoskeleton are autonomy, lightness, and robustness. Such exoskeleton is autonomous and light because it does not contain any actuators and sources of energy. In the 
paper [25], we have investigated a similar passive exoskeleton, but without feet (with pointed feet). Here we take into account the feet - massless feet. From human data the assumption of massless feet is reasonable because its scaling factor for mass is almost equal to $1 \%$ [26].

If we take into account the feet (instead of the pointed feet as in [25]), our model of human walking becomes more realistic (but also much more complex). Using this model with feet, we confirm the result of the paper [25] that during the load transport the human with exoskeleton spends less energy than human alone.We also see in our calculations that the energy consumption of the biped with the feet is more than of the biped without feet (with pointed feet).

In this paper, the proposed study is based on the ballistic movements in the singlesupport and impulsive torques in the instantaneous double-support. The walking in the sagittal plane is studied. We consider the locking of the swing leg knee of the exoskeleton (and as a consequence of the biped) at the instant when this leg touches the bearing surface (becomes support leg) and the unlocking of the knee of the support leg at the instant when it leaves the bearing surface (becomes swing leg). The energy consumption of the impulses is calculated to explore the possibilities to reduce the energy consumption of a biped (human) using an exoskeleton.

Human motions comprise alternating periods of muscle activity and relaxation. The double-support phase is relatively short with respect to the single-support phase and most efforts of the person during walking focused in this double-support phase. Then it is logical to consider the problem of purely ballistic swing phases and instantaneous double-support phases with impulsive inter-link torques. A similar statement of the problem is proposed by Formalskii [27], [28], [29], [30], Mochon and McMahon [31], McGeer [32], and Aoustin and Formalskii [33]. This statement of the problem is mathematically reduced to the boundary value problem with given boundary configurations of the biped.

We consider a 2D model of human to focus the attention to the transport of the payload during a walking. It is a planar anthropomorphic bipedal model with a torso, two identical legs with feet. Similar models are studied in [34] and [35]. The main dynamic effects involved in the motion process of human seems to be based on two important points: the role of gravity and the limited torque available at the ankle. Thus a simplified inverted pendulum model has been used since long time to study walking [36], running [37] and [38], dynamic balance [39]. While a concentrated mass is used to include the role of gravity, the tip of the pendulum corresponds to the punctual contact with the ground or the zero moment point $(Z M P)$ when a flat-footed contact is modeled. Model of human with massless feet has in the single-support phase five DOF and it is described by essentially nonlinear differential equations of tenth order. The mathematical model for the double-support phase is quite different. Thus, the structure of the mathematical model changes during the alternating single- and double-support phases. The exoskeleton has the same DOF, the same size and shape as the biped. The torso, thighs and shins of the exoskeleton are assumed strongly strapped respectively to the torso, thighs and shins of human. In our study, the mass of the exoskeleton is also taken into account.

In this paper, initially we find a ballistic walking of the biped alone (without exoskeleton) for the transport of a payload. The energy consumption of the biped is calculated. After, the biped equipped with the exoskeleton is studied. We find for this biped ballistic walking with the locked knee of the support leg. Then we show that 
the energy consumption for the transport of the same payload is less for the biped equipped with the exoskeleton than for the biped alone.

The impulsive control torques, that are applied in the inter-link joints between the neighboring single-support phases are described by delta-functions of Dirac. Our approach can be considered as asymptotic because it is not possible to carry out these purely impulsive control torques.

The rest of the paper is organized as follows. Section 2 is devoted to the mathematical model of the biped alone and with its walking assist device. The physical parameters of the system are presented here. Also the influence of the massless feet is discussed. The problem definition of the ballistic swing motion is given in Section 3. The boundary configurations and mathematical model of the single-support motion are described in this section. The excursion of the centre of pressure is discussed. The boundary value problem to design ballistic swing motion is also presented here. The instantaneous double-support phase is described through algebraic equations in Section 3. In Section 4, the chosen effort cost functional for the control is described. In Section 5, the results of simulation are shown. Our conclusion and perspectives are offered in Section 6. Appendix contains explicit derivation of the formulas to evaluate the energy consumption with the applied impulsive torques.

\section{Mathematical model of biped alone or equipped with exoskeleton}

Here, we present mechanical model of the biped with feet. Also physical parameters of the biped and the exoskeleton are provided. Mathematical models of the biped alone and with exoskeleton are developed. The influence of the massless feet is described.

\subsection{Physical parameters of the biped and exoskeleton}

For the bipedal model, shown in Figs. 1, we use the physical parameters from [28]. The whole mass of the biped is $75 \mathrm{~kg}$, its height is $1.75 \mathrm{~m}$. The distance between the kneejoint and the centre of mass of the shin is: $s_{s}=0.324 \mathrm{~m}$, between the hip-joint and the centre of mass of the trunk: $s_{T}=0.386 \mathrm{~m}$ (see Fig. 1 (b)). The distance between the hip-joint and the centre of mass of the thigh is: $s_{t}=0.18 \mathrm{~m}$. The head mass is included in the trunk that its length is $l_{T}$. The shape and the degrees of freedom of the exoskeleton are identical to human.

Table 1 gathers the masses, the lengths and the inertia moments for the links of the bipedal model and the walking assist device. Inertia moments are defined with respect to the hip-joint for the trunk and thighs and to the knee-joint for the shins. The parameters are based on the wearable assist device Honda [40].

2.2 Mathematical model of biped with feet

Vector $\mathbf{x}$ of the generalized coordinates for the biped with massless feet is such as

$$
\mathbf{x}=\left[q_{1}, q_{2}, q_{3}, q_{4}, q_{5}, x, y\right]^{\top} .
$$

Here angles $q_{1}, q_{2}, q_{3}, q_{4}$ define the absolute orientation of the shin and thigh for both legs, see Fig. 1 (a). The absolute orientation of the trunk is defined through angular 


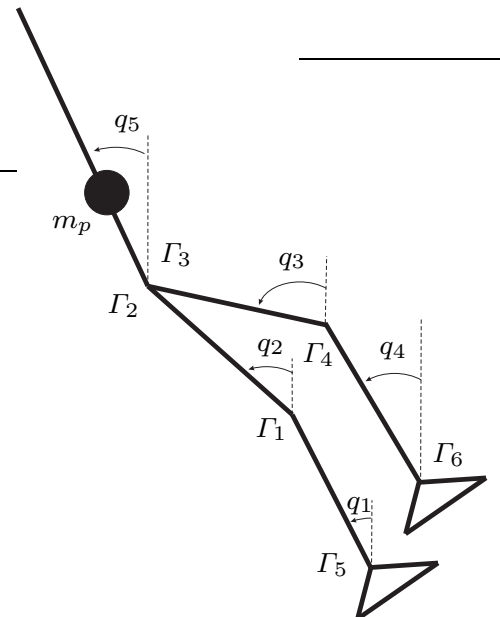

a)

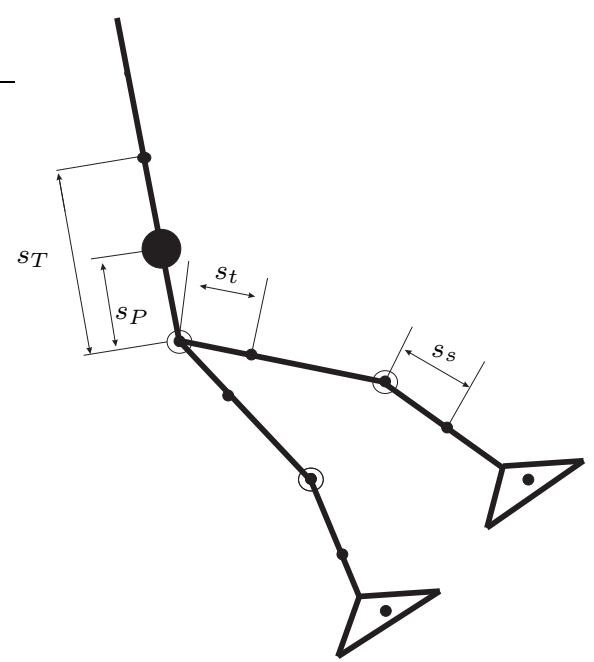

b)

Fig. 1 a) The kinematic model, generalized coordinates, and inter-link torques of human (alone or equipped by exoskeleton); notation $m_{p}$ means mass of the payload. b) Positions of the centres of mass of the links; $s_{P}$ is the distance between the hip-joint and the payload. In our calculations we use the payload with mass $m_{p}=40 \mathrm{~kg}$ and distance $s_{P}=0.1 \mathrm{~m}$.

\begin{tabular}{|c|c|c|c|c|}
\hline & Mass $(\mathrm{kg})$ & Length $(\mathrm{m})$ & $\begin{array}{c}\text { Inertia moment } \\
\left(\mathrm{kg} \cdot \mathrm{m}^{2}\right)\end{array}$ & $\begin{array}{c}\text { centre of } \\
\text { mass }(\mathrm{m})\end{array}$ \\
\hline Human shin & $m_{s}=4.6$ & $l_{s}=0.55$ & $I_{s}=0.0521$ & $s_{s}=0.324$ \\
\hline Human thigh & $m_{t}=8.6$ & $l_{t}=0.45$ & $I_{t}=0.75$ & $s_{t}=0.18$ \\
\hline Human trunk & $m_{T}=48.6$ & $l_{p}=0.75$ & $I_{T}=11.3$ & $s_{T}=0.386$ \\
\hline Exoskeleton shin & $m_{1}=1.0$ & $l_{1}=0.55$ & $I^{1}=0.0260$ & $s_{1}=0.27$ \\
\hline Exoskeleton thigh & $m_{2}=2.0$ & $l_{2}=0.45$ & $I^{2}=0.0354$ & $s_{2}=0.225$ \\
\hline Exoskeleton trunk & $m_{3}=8.0$ & $l_{3}=0.75$ & $I^{3}=0.3817$ & $s_{3}=0.375$ \\
\hline
\end{tabular}

Table 1 Physical parameters of the biped and of the exoskeleton.

variable $q_{5}$. Cartesian hip-joint coordinates are indicated as $x$ and $y$. Superscript ${ }^{\top}$ means transposition.

The mathematical model of the biped alone or with its walking assist device is:

$$
\mathbf{A}(\mathbf{x}) \ddot{\mathbf{x}}+\mathbf{h}(\mathbf{x}, \dot{\mathbf{x}})=\mathbf{D} \boldsymbol{\Gamma}+\mathbf{J}_{\mathbf{r}_{1}}^{\top} \mathbf{r}_{1}+\mathbf{J}_{\mathbf{r}_{2}}^{\top} \mathbf{r}_{2}
$$


Here $\mathbf{A}(\mathbf{x})$ is $7 \times 7$ symmetric positive definite inertia matrix, $\mathbf{h}(\mathbf{x}, \dot{\mathbf{x}})$ is $7 \times 1$ vector, which groups the centrifugal, Coriolis, and gravity forces. $\boldsymbol{\Gamma}$ is $6 \times 1$ vector of the joint torques applied by human or by the brake devices of the exoskeleton. We consider six torques applied in the hip-, knee- and ankle-joints. Vectors $\mathbf{r}_{i}$, with $i=1,2$, are the wrench ground reactions applied to the massless feet (and consequently to the ankle-joints). Vector $\mathbf{r}_{i}=\left(r_{i x}, r_{i y}\right)^{\top}$ is composed of the horizontal $r_{i x}$ and vertical $r_{i y}$ components of the ground reaction.

The following constraint equations are correct when the front or/and rear leg is/are on the bearing surface.

$$
\mathbf{J}_{\mathbf{r}_{i}} \ddot{\mathbf{x}}+\dot{\mathbf{J}}_{\mathbf{r}_{i} \dot{\mathbf{x}}=\mathbf{0}} \quad \text { for } i=1 \text { or } / \text { and } 2 .
$$

We apply the principle of virtual work to calculate matrix $\mathbf{D}$ in equation (2), see [41]. The joint variables $\theta_{i}$ for $i=(1, \cdots, 6)$ as functions of the generalized coordinates are as follows:

$$
\begin{gathered}
\theta_{1}=q_{2}-q_{1}, \quad \theta_{2}=q_{5}-q_{2}, \quad \theta_{3}=q_{5}-q_{3}, \\
\theta_{4}=q_{3}-q_{4}, \quad \theta_{5}=q_{1}, \theta_{6}=q_{4} .
\end{gathered}
$$

Variables $\theta_{i}(i=1,2,3,4)$ are calculated up to value $\pi$.

The virtual work $\delta W_{i}$ of each torque $\Gamma_{i}$, applied to the corresponding joint with virtual variable $\delta \theta_{i},(i=1, \cdots, 6)$ is described by equation:

$$
\delta W_{i}=\Gamma_{i} \delta \theta_{i}=\Gamma_{i} \mathbf{D}_{i}^{\top} \delta \mathbf{x}
$$

Then matrix $\mathbf{D}$ of the torques is: $\mathbf{D}=\left[\mathbf{D}_{1}, \mathbf{D}_{2}, \mathbf{D}_{3}, \mathbf{D}_{4}, \mathbf{D}_{5}, \mathbf{D}_{6}\right]$ with the following columns,

$$
\mathbf{D}_{i}=\frac{\partial}{\partial \Gamma_{i}}\left(\frac{\partial \delta W}{\partial \delta \mathbf{x}}\right)
$$

If the torques $\Gamma_{i},(i=, 1, \cdots, 6)$ from human or exoskeleton are applied in these six joints, matrix $\mathbf{D}$ is as follows:

$$
\mathbf{D}=\left(\begin{array}{cccccc}
-1 & 0 & 0 & 0 & 1 & 0 \\
1 & -1 & 0 & 0 & 0 & 0 \\
0 & 0 & -1 & 1 & 0 & 0 \\
0 & 0 & 0 & -1 & 0 & 1 \\
0 & 1 & 1 & 0 & 0 & 0 \\
0 & 0 & 0 & 0 & 0 & 0 \\
0 & 0 & 0 & 0 & 0 & 0
\end{array}\right)
$$

The principle of virtual work can be also used to obtain matrices $\mathbf{J}_{\mathbf{r}_{1}}$ and $\mathbf{J}_{\mathbf{r}_{2}}$.

\subsection{Influence of the massless feet}

The orientation of the massless foot of the transferred leg (in the single-support) can be instantaneously changed and this foot does not influence the dynamics of the biped (alone or with exoskeleton) in the single-support.

During the single-support motion, the flat massless foot of the support leg $i$ is in the equilibrium on the ground. In Fig. 2, applied to the foot the ground reaction 
with horizontal $r_{i x}$ and vertical $r_{i y}$ components is shown; $l_{x}$ and $l_{y}$ are respectively horizontal and vertical components of the distance between the ankle-joint and the point of ground reaction application - centre of pressure COP. The moment around the axis passing through this point orthogonally to the sagittal plane is null. Furthermore, this point remains inside the bearing surface, with the foot flat on the floor, and there is no tipping of the support foot [42]. We do not take into account the weight of the foot because according to our assumption the feet are massless. The moment $M$ around the ankle-joint of the ground reaction force applied to the foot is the following:

$$
M=r_{i y} l_{x}+r_{i x} l_{y} .
$$

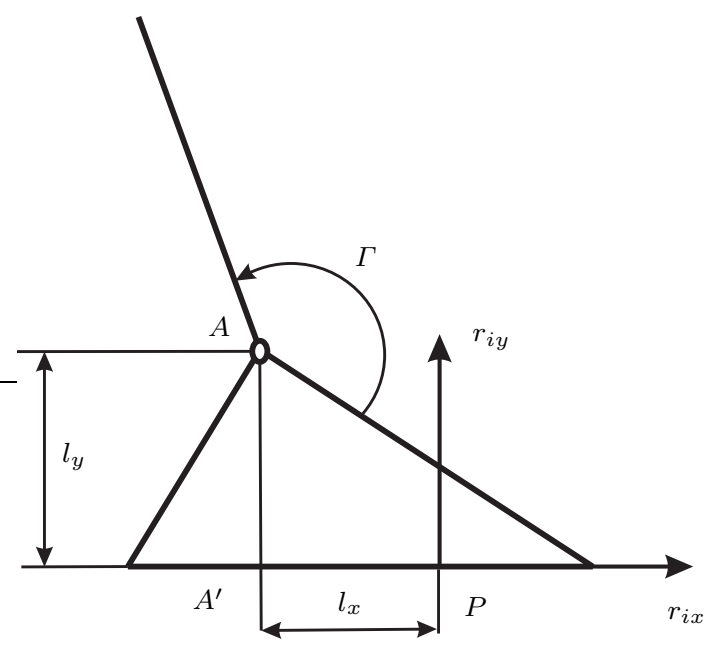

Fig. 2 Horizontal $r_{i x}$ and vertical $r_{i y}$ components of the ground reaction; horizontal $l_{x}$ and vertical $l_{y}$ components of the distance between the ankle-joint and $C O P$; moment $\Gamma$ applied by the human to the shin in the ankle-joint $A$ of the support leg.

The conditions of the equilibrium of the flat massless foot of the support leg are conditions of its static balance. According to these conditions, the force $-\mathbf{r}_{i}$ with horizontal $-r_{i x}$ and vertical $-r_{i y}$ components and also moment

$$
-M=-r_{i y} l_{x}-r_{i x} l_{y}
$$

have to be applied (in the clockwise direction) in the ankle-joint to the massless support foot. Consequently the force $\mathbf{r}_{i}$ is applied to the shin of the support leg in the ankle-joint A. Also the torque:

$$
\Gamma=r_{i y} l_{x}+r_{i x} l_{y}
$$

has to be applied (in the counterclockwise direction) by human in the ankle-joint $A$ to the shin of the support leg (see Fig. 2).

In paper [25], we used the same (as for the biped with massless feet) vector (1) of the generalized coordinates to describe the biped without feet (with pointed feet). The mathematical model is also the same (see matrix equation (2)). But the size of vector 
$\boldsymbol{\Gamma}$ are $4 \times 1$, because with pointed feet the torques in the ankle-joints are $\Gamma_{5}=\Gamma_{6}=0$. And in double-support for a biped with pointed feet it is possible to apply torques only in the hip- and knee-joints of both legs.

\section{Ballistic swing motion in single-support phase: problem definition}

The biped walking consists of the alternating phases of single- and double- support. In the single-support motion, one of the legs is support leg; it is placed on the bearing surface - on the ground. But the other leg is the swing one. The single-support motion of the biped is modeled in this paper by a ballistic (passive) one. The problem of design of the ballistic motion is reduced mathematically to the boundary-value problem for the system of nonlinear differential equations with given initial, final configurations and the duration of the step. In this section, we describe way to design ballistic swing motion. The ways to design ballistic motion are similar for the biped alone and for the biped equipped by the exoskeleton.

\subsection{Boundary configurations, mathematical model}

It is assumed that in the single-support phase, there are no sliding motion and no take-off of the support leg (leg 1) of the biped alone and equipped by exoskeleton. Furthermore, with a torque $\Gamma_{1}$, the exoskeleton carries out the locking of the knee of this support leg by any mechanical brake (without any motor).

Let $\mathbf{x}(0)$ be the initial configuration of the biped at time $t=0$. The final configuration of the biped in the single-support phase at the given time $t=T$ is noted $\mathbf{x}(T)$. In Fig. 3, both boundary configurations $\mathbf{x}(0)$ and $\mathbf{x}(T)$ are shown as side view. The left pose in Fig. 3 is the initial configuration $\mathbf{x}(0)$, the right pose is the terminal configuration $\mathbf{x}(T)$. Our biped is walking from left to right. The final configuration is similar to the initial configuration with the legs swapped. Both legs are straightened in these boundary configurations, the torso is slightly inclined forward $\left(q_{5}(0)=-3^{\circ}\right)$. We assume, the front and hind legs in the initial configuration are respectively the support and swing legs. The support leg is shown by solid line, the swing leg - by dashed line.

In real human walking, the double-support motion is distributed in time, and consequently the configuration of the human at the beginning of the double-support (at the end of previous single-support motion) differs from the configuration at the end of this double-support (at the beginning of the next single-support motion). Consequently for the human walking the configurations at the beginning and at the end of the single-support motion are different. We consider model with instantaneous doublesupport phase, and in our model, the configuration of the biped at the end of the double-support has to be the same as at the start of this phase. We have chosen the boundary configurations with straightened legs. In our opinion, the boundary configurations with straightened legs are not far from the human-like configurations at the beginning and at the end of single-support motion.

It appears, that the human with passive exoskeleton (without any drives) tries to walk with its usual gate. Therefore, we use in our investigations the same boundary configurations for the biped alone and equipped with the exoskeleton.

Let $L$ be the length of the step corresponding to a single-support. 


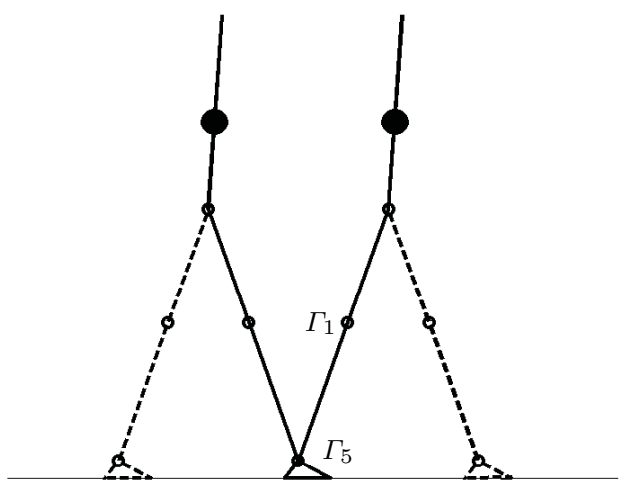

Fig. 3 Initial $\mathbf{x}(0)$ and final $\mathbf{x}(T)$ configurations of the biped (side view).

We consider a ballistic motion during the single-support phase on the support leg 1 . The torques provided by the biped (human) are null except at the ankle of the support leg, then $\Gamma_{5} \neq 0$ and $\Gamma_{i}=0$, with $i=2,3,4$, and 6 . As a consequence, the matrix equation (2) for the ballistic motion becomes:

$$
\mathbf{A}(\mathbf{x}) \ddot{\mathbf{x}}+\mathbf{h}(\mathbf{x}, \dot{\mathbf{x}})=\mathbf{D}\left[\begin{array}{c}
\Gamma_{1} \\
\mathbf{0}_{3 \times 1} \\
\Gamma_{5} \\
0
\end{array}\right]+\mathbf{J}_{\mathbf{r}_{1}}^{\top} \mathbf{r}_{1}
$$

with the constraint equation (3) for the support leg foot fixed on the ground:

$$
\mathbf{J}_{\mathbf{r}_{1}} \ddot{\mathbf{x}}+\dot{\mathbf{J}}_{\mathbf{r}_{1} \dot{\mathbf{x}}}=\mathbf{0}
$$

When we consider the motion of the biped alone (without exoskeleton), then also equation

$$
\Gamma_{1} \equiv 0
$$

has to be added because the support leg of the biped alone is not locked. But when the motion of the biped equipped by exoskeleton is considered, then the constraint equation for the knee of the support leg locked (in the swing phase) has to be added (instead of equation (13)):

$$
\theta_{1}=q_{2}-q_{1} \equiv 0
$$

and torque $\Gamma_{1}$ becomes unknown variable. Notation $\mathbf{0}_{3 \times 1}$ in equation (11) means the column with three zero-elements. Each of systems (11), (12), (13) and (11), (12), (14) contains 10 scalar equations but with 11 unknown variables: $q_{1}, q_{2}, q_{3}, q_{4}, q_{5}, x, y, \Gamma_{1}$, $\Gamma_{5}, r_{1 x}$, and $r_{1 y}$. In both cases, we consider a supplementary equation (10) for torque $\Gamma_{5}$ in the ankle-joint of the massless support foot. 
3.2 Excursion of centre of pressure (COP)

Equation (10) can be used if the progression $l_{x}$ of the $C O P$ along the sole during the single-support phase is prescribed as function of time $l_{x}=l_{x}(t / T)(T$ is the last instant of the single-support motion). Previous researches have modeled $l_{x}$ as linear function of time, [28]; [43]. However, experiments [44]; [45] reveal that this function is a more complex one, involving at least polynomial expressions of higher order. Furthermore, Lugade and Kaufman [45] demonstrated that the COP excursion was from the heel, but not exactly to the toe. Therefore, the chosen progression of the COP is defined from the heel defined by abscissa $l_{x}(0)=-l_{f}$ to a point that is defined by abscissa $l_{x}(1)=L_{f}$ such as (see Figure 4 for the foot on support):

$$
l_{x}=-l_{f}+\psi(t / T)\left(L_{f}+l_{f}\right),
$$

with $\psi(0)=0, \psi(1)=1$.

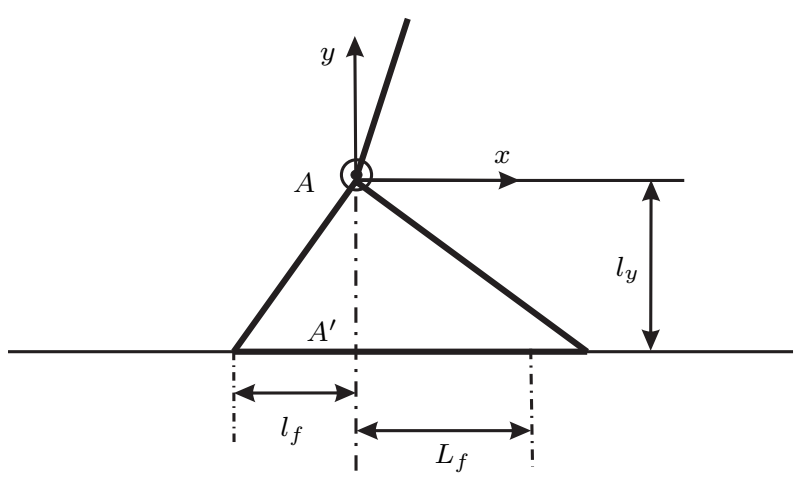

Fig. 4 Size characteristics of the foot: $l_{f}$ is the distance between the heel and the projection on the sole of the ankle-joint $A, L_{f}$ is the distance between this projection and $C O P$ at the last instant of the single-support motion $t=T$.

Value $L_{f}$ is less than the distance between the projection on the sole of the anklejoint and the toe (see Fig. 4). We use the numerical data $l_{f}=0.04 m, L_{f}=0.07 \mathrm{~m}$ and $l_{y}=0.07 \mathrm{~m}$ for all our numerical tests. With these values there is no take-off in the single-support nor at the impact of the support legs. According to observations of Lugade and Kaufman [45] function $\psi(t / T)$ is chosen as a polynomial function of fourth order as follows:

$$
\psi(t / T)=a_{0}+a_{1} \frac{t}{T}+a_{2}\left(\frac{t}{T}\right)^{2}+a_{3}\left(\frac{t}{T}\right)^{3}+a_{4}\left(\frac{t}{T}\right)^{4} .
$$

The five coefficients $a_{i}, i=0, \cdots, 4$ are calculated such that $\psi(0)=0, \psi(1)=1$, $\dot{\psi}(0)=\dot{\psi}(1)=0$, and $\psi(1 / 2)=1 / 2$. Figure 5 represents this polynomial function (16). Function $\psi(t / T)$, along with the formula (15), defines the distance $l_{x}$ as function of time $l_{x}=l_{x}(t / T)$. Using functions (15), (16) we can define the torque $\Gamma_{5}$ at the ankle of the support leg 1 as follows:

$$
\Gamma_{5}=l_{x} r_{1 y}+l_{y} r_{1 x}
$$




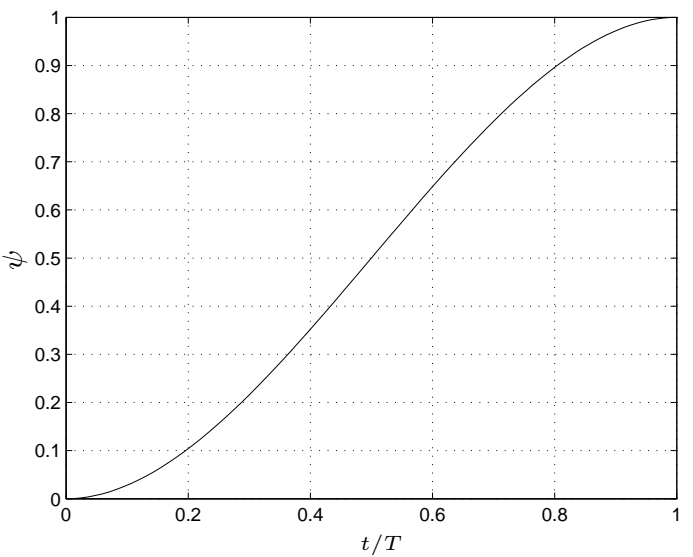

Fig. 5 Profil of function $\psi(t / T)$.

\subsection{Boundary value problem}

The dynamic behavior of the biped is defined through equations (11) - (17). Substituting expression (17) into equation (11), we can write the following matrix equation:

$$
\mathbf{A}(\mathbf{x}) \ddot{\mathbf{x}}+\mathbf{h}(\mathbf{x}, \dot{\mathbf{x}})=\mathbf{D}\left[\begin{array}{c}
\Gamma_{1} \\
\mathbf{0}_{5 \times 1}
\end{array}\right]+\left[\begin{array}{ll}
\mathbf{D}_{5} l_{y}+\mathbf{J}_{\mathbf{r}_{11}}^{\top} & \mathbf{D}_{5} l_{x}+\mathbf{J}_{\mathbf{r}_{12}}^{\top}
\end{array}\right] \mathbf{r}_{1}
$$

where $\mathbf{J}_{\mathbf{r}_{11}}^{\top}$ and $\mathbf{J}_{\mathbf{r}_{12}}^{\top}$ are sub-matrices such that $\mathbf{J}_{\mathbf{r}_{1}}^{\top}=\left[\mathbf{J}_{\mathbf{r}_{11}}^{\top}(7 \times 1), \quad \mathbf{J}_{\mathbf{r}_{12}}^{\top}(7 \times 1)\right]$.

The ballistic single-support motion of the biped alone (without exoskeleton) is described by matrix equations (12), (13), (15) - (18). The numerical values of the parameters in matrices $\mathbf{A}(\mathbf{x}), \mathbf{h}(\mathbf{x}, \dot{\mathbf{x}})$, and $\mathbf{J}_{\mathbf{r}_{1}}$ of course are different for the mathematical models of the biped with exoskeleton and without exoskeleton.

To design the ballistic motion of the biped alone in the single-support phase, it is necessary to find solution $\mathbf{x}(t)$ of the equations (12), (13), (15) - (18) with the given boundary conditions $\mathbf{x}(0)$ and $\mathbf{x}(T)$ and time $T$. To design the ballistic motion of the biped with the exoskeleton it is necessary to find solution $\mathbf{x}(t)$ of matrix equations (12), (14)-(18) with the same given boundary conditions and time $T$. In each case, we have to find the initial velocity vector $\dot{\mathbf{x}}(0)$ such that solution $\mathbf{x}(t)$, starting from the given initial configuration $\mathbf{x}(0)$ with the velocity vector $\dot{\mathbf{x}}(0)$, reaches the given final configuration $\mathbf{x}(T)$ at the given time $T$. Unknown velocity vector $\dot{\mathbf{x}}(0)$ for the biped with the exoskeleton must satisfy condition $\dot{\theta}_{1}(0)=\dot{q}_{2}(0)-\dot{q}_{1}(0)=0$ (see also identity (14)).

The given boundary conditions $\mathbf{x}(0)$ and $\mathbf{x}(T)$ are chosen such that the positions of the biped with its exoskeleton are similar to human configurations. The formulated above boundary value problem can be numerically solved using a Newton method with vector $\dot{\mathbf{x}}(0)$ unknown. The motion of the biped is admissible, if the vertical component of the ground reaction in the support leg is positive (directed upwards), if the swing leg moves over the ground for $0<t<T$, and if the swing leg bends the knee forward. We check these constraints after solving the boundary value problem - a posteriori. The 
ground reaction $\mathbf{r}_{1}$ and the torque $\boldsymbol{\Gamma}_{1}$ developed by the brake device are calculated during solving this boundary value problem with matrix equations (12)-(18).

In the single-support motion, the Cartesian coordinates $x$ and $y$ of the hip-joint depend on the interlink angles of the stance leg. Therefore, in fact, in our boundary value problem for biped alone, we search five initial unknown angular velocities in five interlink joints to reach the five given angles in theses five interlink joints. Due to identity (14), for the biped with exoskeleton we have to find four initial unknown angular velocities in four interlink joints to reach the four given angles in theses four interlink joints. So, the number of unknown variables is equal to the number of the equations. The existence of the solution of the boundary value problem is analytically proved ( [27] - [30]) for linearized mathematical models of the biped. But it is possible also to find numerically solutions of the nonlinear boundary value problem for many realistic parameters of biped walking - time, length of the step etc ( [27] - [30]). In this paper, we also numerically find the solution of nonlinear boundary value problem for biped alone and equipped with exoskeleton. For the biped alone this solution is such that the transferred leg moves above the support and bends the knee forward, the stance leg bends slightly in the knee and remains almost straight all the time, the torso makes just one oscillation close to the vertical axis with small amplitude, and the reaction force is directed upwards. We do not prescribe all these features beforehand in the statement of the problem, but observe them after solving the boundary value problem. So, the ballistic locomotion of our biped looks human-like gait. To get this solution of the boundary value problem numerically we use the fsolve function based on the Newton-Raphson Algorithm of Matlab $($ ).

After solving the boundary value problem, the vector of the initial velocities $\dot{\mathbf{x}}(0)$ becomes known. We denote it by $\dot{\mathbf{x}}^{a}$. If the initial conditions $\mathbf{x}(0), \dot{\mathbf{x}}^{a}$ are known, then by integration of the system (12), (13), (15) - (18) (for biped alone) or of the system (12), (14)-(18) (for biped with exoskeleton) the vector of the terminal velocities $\dot{\mathbf{x}}(T)$ can also be found. We denote it by $\dot{\mathbf{x}}^{b}$.

During described above single-support motion of the biped alone, the torque in each joint is null except at the ankle of the support leg. In this ankle-joint, torque (17) is applied to the shin. But, we call this motion as ballistic because there are no torques in the other five joints (remember that the feet are massless). During singlesupport motion of the biped with the exoskeleton there is also a torque to lock the knee of the support leg. But, we also call this motion as ballistic because this knee is locked by mechanical brake and there are no torques in the knee- and ankle-joint of the transferred leg and in the hip-joints.

\subsection{Instantaneous double-support phase with impulses}

In the human gait the time of the double-support is less than $20 \%$ of the whole step period. Some researchers (see for example Mochon and McMahon [31]) suppose that during human walking, most of the efforts take place in the double-support phase. Taking into account just these considerations, we study locomotion with ballistic swing phases and instantaneous double-support phases. It means that at the instant of the double-support, the ground reaction forces and the torques applied in the joints of our biped with and without exoskeleton should be impulsive efforts and described by Dirac delta-functions. This approach can be considered as an asymptotic one because the impulsive efforts, describing by delta-functions, cannot be realized. At the instant 
of double-support phase the configuration of the biped does not change. It means particularly that there is no sliding of the legs at this phase. In the case where the ground reaction force applied to the leg has finite values, there is no sliding of this leg, only if the ground reaction vector is situated in the cone of friction [46]. Therefore, we have adopted the assumption that the impulsive ground reaction force should be in the cone of friction. The critical value of the friction coefficient (angle of friction cone) can be found after calculation of the magnitudes of the impulsive horizontal and vertical components of the ground reaction.

During the biped's gait, an impact occurs at the end of the single-support phase, when the swing leg touches the ground. If we assume that there are not any active torques applied in the joints and after impact this leg remains on the support, then we have to conclude that the inelastic passive impact occurs. At the instant of such an impact, the biped looses energy. Therefore, the velocity vector after the impact will not be the desired one, if the bearing surface is horizontal. Then for the next ballistic step the desired initial velocity vector will not be reached. As a consequence, a complete walking cyclic gait of the biped alone or with its assist device cannot be realized on a horizontal surface without active torques.

At the instant of impact, denoted by $T$, the double-support phase is assumed instantaneous. However, theoretically, "around" the instantaneous double-support it is possible to define impulsive torques in order to ensure the desired velocity jump; see Formalskii [28], [29], [30], Hurmuzlu, and Chang [47]. These torques have to be developed by the biped.

The relations to find control impulsive torques in the double-support phase are obtained from the general mathematical model (2), (3).

Similarly to [29,30,48] or [33] let us consider the current ballistic motion on the support leg 1 and the following ballistic motion on the support leg 2. The swing leg 2 touches the ground just after the end of the ballistic single-support motion on the support leg 1 , and an impact occurs. The initial and final velocity vectors $\dot{\mathbf{x}}^{a}$ and $\dot{\mathbf{x}}^{b}$ of the ballistic swing motion are known from the solution of the boundary value problem for the biped alone and with the exoskeleton. Let us consider the instantaneous doublesupport phase with impulsive forces between two neighboring single-support phases (see Fig. 6). Recall that the role of both legs exchanges after the end of the single-support motion on the support leg 1 . In the next single-support motion, support leg 1 becomes the transferring leg and the transferring leg 2 becomes the support leg.

Passive impact with the ground

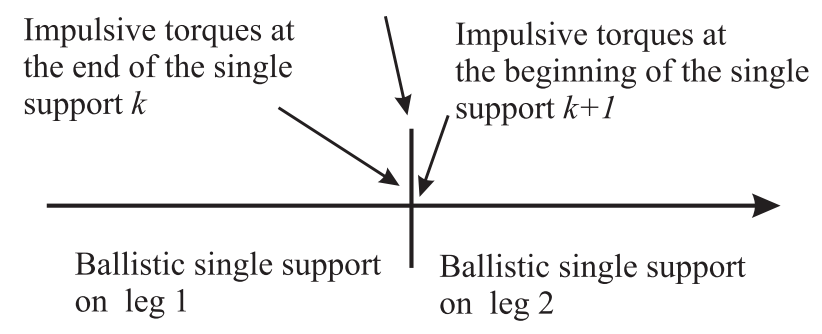

Fig. 6 Decomposition of the instantaneous double-support phase; see [48], [30], or [33]. 
At this instantaneous double-support phase simultaneously the leg 2 lands on the ground and the leg 1 takes off. We divide this instantaneous double-support phase into three instantaneous sub-phases and investigate them. Just before the passive impact with the ground (at the first sub-phase), let us apply the impulsive torques in the joints

$$
\boldsymbol{\Gamma}(t)=\mathbf{I}^{-} \delta\left(t-T^{-}\right)
$$

with the vector of the magnitudes $\mathbf{I}^{-}\left(I_{1}^{-}, I_{2}^{-}, I_{3}^{-}, I_{4}^{-}, I_{5}^{-}, 0\right)$. In formula (19), $\delta\left(t-T^{-}\right)$ is Dirac delta-function which is not zero at the instant $t=T^{-}$of the first sub-phase. Just after the passive impact with the ground (at the time-instant $t=T^{+}$of the third sub-phase), let us apply the following impulsive torques in the joints

$$
\boldsymbol{\Gamma}(t)=\mathbf{I}^{+} \delta\left(t-T^{+}\right)
$$

with the vector of the magnitudes $\mathbf{I}^{+}\left(I_{1}^{+}, I_{2}^{+}, I_{3}^{+}, I_{4}^{+}, 0, I_{6}^{+}\right)$.

Now we will consider each sub-phase in more details.

- The first sub-phase is the time-instant just after the end of the ballistic singlesupport motion on the support massless foot 1 and just before contact with the ground of the swing massless foot 2 (before passive impact). In the first sub-phase (we denote time-instant of this sub-phase as $T^{-}$), impulsive torques (19) are applied. All torques are developed by the human, if there is no exoskeleton. If the human is equipped with the exoskeleton, the knee of the leg 1 (hind leg) is kept locked through an impulsive torque $\Gamma_{1}(t)=I_{1}^{-} \delta\left(t-T^{-}\right)$developed by the brake device mounted in this knee of the exoskeleton. At the same instant $T^{-}$, the impulsive ground reaction $\mathbf{r}_{1}(t)=\mathbf{I}_{\mathbf{r}_{1}}^{-} \delta\left(t-T^{-}\right)$is applied in the stance leg tip. Here $\mathbf{I}_{\mathbf{r}_{1}}^{-}\left(I_{\mathbf{r}_{1 x}}^{-}, I_{\mathbf{r}_{1 y}}^{-}\right)$is the vector of the magnitudes of the impulsive ground reaction in the stance leg 1 . Under the impulsive torques, the velocity vector $\dot{\mathbf{x}}$ of the biped changes instantaneously from the value $\dot{\mathbf{x}}^{b}$ to some value $\dot{\mathbf{x}}^{-}$(unknown). The corresponding equations for the jump of velocities can be obtained through the integration of the general equations of motion (2) for the infinitesimal time from $T^{-}$ to $T$ such as:

$$
\mathbf{A}[\mathbf{x}(T)]\left(\dot{\mathbf{x}}^{-}-\dot{\mathbf{x}}^{b}\right)=\left[\begin{array}{llllll}
\mathbf{D}_{1} & \mathbf{D}_{2} & \mathbf{D}_{3} & \mathbf{D}_{4} & \mathbf{D}_{5} & \mathbf{D}_{6}
\end{array}\right] \mathbf{I}^{-}+\mathbf{J}_{\mathbf{r}_{1}}^{\top} \mathbf{I}_{\mathbf{r}_{1}}^{-} .
$$

The torques provided by the Coriolis and gravity forces have finite values. Thus, they do not influence the velocity jump. Vector $\mathbf{x}(T)$ describes in matrix equation (21) the configuration of human at the instant of impulsive actions. This configuration $\mathbf{x}(T)$ does not change at the double-support phase i.e. at the instants of the first, second, and third sub-phases. Vector $\dot{\mathbf{x}}^{-}$describes the velocities of the biped after the first sub-phase.

Torque $\Gamma_{5}(t)=I_{5}^{-} \delta\left(t-T^{-}\right)$(see expression (19)) with magnitude $I_{5}^{-}$is applied in the ankle of the stance leg (hind leg) at the instant $t=T^{-}$. It ensures that the $C O P$ is located at abscissa $L_{f}$ under the sole of the massless foot 1 as follows:

$$
I_{5}^{-}=I_{r_{1 y}}^{-} L_{f}+I_{r_{1 x}}^{-} l_{y}
$$

The velocity of the stance leg (hind leg) foot remains zero after the first subphase. Therefore, we have to take into account the following matrix constraint equation:

$$
\mathbf{J}_{\mathbf{r}_{1}} \dot{\mathbf{x}}^{-}=\mathbf{0}_{2 \times 1}
$$


For the human with exoskeleton, the velocity of the inter-link angle in the knee of the stance leg (hind leg) after first sub-phase remains zero through the impulsive brake effort $\Gamma_{1}(t)=I_{1}^{-} \delta\left(t-T^{-}\right)$(see expression (19)), therefore:

$$
\mathbf{D}_{1}^{\top} \dot{\mathbf{x}}^{-}=0 .
$$

Just after first sub-phase the tip of the leg 2 (front leg) is not on the ground still and the velocity vector of this tip has to be directed downwards to the ground. We have to take into account this condition in our calculations.

- At the second sub-phase, human without exoskeleton does not apply any torques in the joints. But if human is equipped with exoskeleton, then the knee of the leg 2 (front leg) is mechanically locked by the brake device and the knee of the leg 1 (hind leg) is unlocked. We call this second sub-phase "passive impact", because at the instant of this sub-phase, there are no torques applied by the human. We assume this passive impact as absolutely inelastic. The time-instant of this passive impact is $t=T$. Given these conditions, the locking torque provided by the exoskeleton can be considered as impulsive $\Gamma_{4}(t)=I_{4} \delta(t-T)$; the ground reaction at this instant of an impact also can be defined by the delta-function $\mathbf{r}_{2}(t)=\mathbf{I}_{\mathbf{r}_{2}} \delta(t-T)$. Here $I_{4}$ is the magnitude of the impulsive locking torque in the knee of leg 2 (front leg) and $\mathbf{I}_{\mathbf{r}_{2}}\left(I_{\mathbf{r}_{2 x}}, I_{\mathbf{r}_{2 y}}\right)$ is the vector of the magnitudes of the impulsive ground reaction in this leg 2 . The corresponding equations for the velocities jump can be obtained through the integration of the matrix equation (2) for the infinitesimal time:

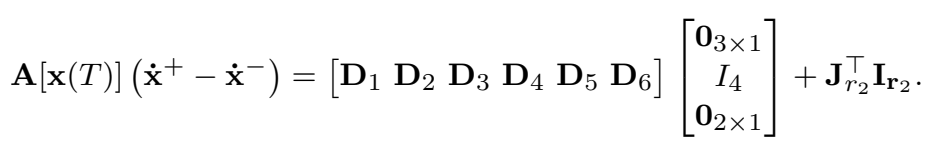

Here $\dot{\mathbf{x}}^{+}$is the velocity vector (unknown) just after an inelastic passive impact.

The velocity of the inter-link angle in the knee of the stance leg (front leg) after the second sub-phase remains zero through the impulsive brake effort $\Gamma_{4}(t)=$ $I_{4} \delta(t-T)$, therefore

$$
\mathbf{D}_{4}^{\top} \dot{\mathbf{x}}^{+}=0
$$

The swing leg 2 (front leg) after the impact becomes a stance leg. Therefore, its tip velocity becomes zero after the impact,

$$
\mathbf{J}_{\mathbf{r}_{2}} \dot{\mathbf{x}}^{+}=\mathbf{0}_{2 \times 1} \text {. }
$$

The velocity of the stance leg tip 1 (hind leg) before an impact is equal to zero. After the instantaneous passive impact the ground reaction in leg 1 has to be zero and the velocity vector of the leg 1 tip has to be directed upwards - from the ground. We have to take into account this condition in our calculations.

- Now let us consider the third sub-phase. The swing leg 1 (hind leg) takes off the ground at the second sub-phase, which is the passive impact. Then, the next ballistic single-support motion on leg 2 (front leg) starts. However, before the next ballistic swing motion (just after the take-off of the leg 1), in the third sub-phase at timeinstant $t=T^{+}$, impulsive torques (20) are applied. If there is no exoskeleton, then all torques are applied by human. If the human is equipped with the exoskeleton, then the torque in the knee of the front leg (leg 2) is developed by the brake device of the exoskeleton and the locking of this knee is kept through the impulsive torque $\Gamma_{4}(t)=I_{4}^{+} \delta\left(t-T^{+}\right)$(see expression (20)). The goal of the impulsive torques in the 
third sub-phase is to change the velocity vector of the biped instantaneously from the value $\dot{\mathbf{x}}^{+}$(which takes place just after passive impact) to the known velocity vector $\dot{\mathbf{x}}^{a}$ (which has to be at the start of the next single-support phase).

Integrating the differential equations (2) for the infinitesimal time from $T$ to $T^{+}$we come to the following matrix relation:

$$
\mathbf{A}[\mathbf{x}(T)]\left(\dot{\mathbf{x}}^{a}-\dot{\mathbf{x}}^{+}\right)=\left[\begin{array}{llllll}
\mathbf{D}_{1} & \mathbf{D}_{2} & \mathbf{D}_{3} & \mathbf{D}_{4} & \mathbf{D}_{5} & \mathbf{D}_{6}
\end{array}\right]\left[\begin{array}{c}
\mathbf{I}_{4 \times 1}^{+} \\
0 \\
I_{6}^{+}
\end{array}\right]+\mathbf{J}_{\mathbf{r}_{2}}^{\top} \mathbf{I}_{\mathbf{r}_{2}}^{+} .
$$

Here (see expression (20)) $\Gamma_{i}(t)=I_{i}^{+} \delta\left(t-T^{+}\right)(i=1,2,3$, and 6) are the four impulsive torques applied by the human.

The impulsive torque $\Gamma_{6}(t)=I_{6}^{+} \delta\left(t-T^{+}\right)$, which is applied in the ankle of the front leg at the instant $t=T^{+}$, ensures that the $C O P$ is located at abscissa $-l_{f}$ under the sole of the massless foot 2 (front leg).

$$
I_{6}^{+}=-I_{r_{2 y}}^{+} l_{f}+I_{r_{2 x}}^{+} l_{y} .
$$

Equations (21) - (29) are composed of $\mathbf{2 9}$ scalar equations to find $\mathbf{3 1}$ unknown variables:

$$
\begin{gathered}
\dot{\mathbf{x}}^{-}, \quad I_{1}^{-}, \quad I_{2}^{-}, \quad I_{3}^{-}, \quad I_{4}^{-}, \quad I_{6}^{-}, \quad I_{r_{1 x}}^{-}, \quad I_{r_{1 x}}^{-}, \quad I_{r_{1 y}}^{-}, \quad \dot{\mathbf{x}}^{+}, \\
I_{4}, \quad I_{r_{2 x}}, \quad I_{r_{2 y}}, \quad I_{1}^{+}, \quad I_{2}^{+}, \quad I_{3}^{+}, \quad I_{4}^{+}, \quad I_{r_{2 x}}^{+}, \quad I_{r_{2 y}}^{+} .
\end{gathered}
$$

(Remark: With (22) and (29) the impulsive torques $I_{5}^{-}$and $I_{6}^{+}$respectively depend on $\left(I_{r_{1 x}}^{-}, \quad I_{r_{1 y}}^{-}\right)$and $\left.\left(I_{r_{2 x}}^{+}, \quad I_{r_{2 y}}^{+}\right)\right)$. Then the problem of impulsive control has an infinite number of solutions. But if the number of equations is less than the number of unknown variables, it is possible to extract a unique solution minimizing some cost functional. The components of the above-mentioned vectors are the subjects of the minimization. Among this set of components, $\mathbf{3 1}-\mathbf{2 9}=\mathbf{2}$ can be defined as optimization variable to minimize a cost functional.

Equations (21) - (29), that are used to calculate the impulsive torques at the instantaneous double-support phase for the biped with exoskeleton, are valid also for the biped (human) alone, except equations (24) and (26). Indeed human without exoskeleton does not have any brake devices. We assume that the human without exoskeleton applies himself the impulsive torques in the ankle-, knee-, and hip-joints. Then similarly to the case of the biped with exoskeleton matrix equations (21) - (23), (25), (27) - (29) contains also $\mathbf{2 7}$ scalar equations to find $\mathbf{3 0}$ unknown variables. These unknown variables are the same as in (30) except variable $I_{4}$.

\section{Criteria to evaluate energy consumption}

The choice of a criterion is difficult and subjective. Furthermore it is not sure if some criterion is optimized during a human walking. Nevertheless for the numerical tests, from a physical point of view we consider the following criterion, proposed in [49]. To 
evaluate the energy costs $W_{s s}$ (index "ss" means "single-support") of the biped with massless feet we use the following integral:

$$
\mathbf{W}_{s s}=\int_{0}^{T}\left|\Gamma_{5}(t) \dot{\theta}_{5}(t)\right| \mathrm{d} t
$$

Product $\Gamma_{5}(t) \dot{\theta}_{5}(t)$ in expression (31) is the power of torque $\Gamma_{5}(t)$ (see $(17)$ ). This criterion is based on the mechanical energy provided by human. But we assume that the actuated joints are not regenerative - energy cannot be restored. Therefore, absolute value $\left|\Gamma_{5}(t) \dot{\theta}_{5}(t)\right|$ is used as integrand in expression (31).

Now let us consider the biped alone in the double-support phase. To evaluate the energy consumption $W_{d s}$ (index "ds" means "double-support") of the impulsive torques that are applied at the first and third sub-phases the criterion can be expressed as:

$$
\mathbf{W}_{d s}=\sum_{i=1-5} \int_{T^{-}}^{T}\left|\Gamma_{i}(t) \dot{\theta}_{i}(t)\right| \mathrm{d} t+\sum_{i=1-4,6} \int_{T}^{T^{+}}\left|\Gamma_{i}(t) \dot{\theta}_{i}(t)\right| \mathrm{d} t .
$$

In expression (32) six torques developed by the human in ankle-, knee-, and hipjoints are taken into account. Expression (32) can be written in the following form:

$$
\mathbf{W}_{d s}=\sum_{i=1-5} W_{i}^{-}+\sum_{i=1-4,6} W_{i}^{+}
$$

Values $W_{i}^{-}(i=1,2,3,4,5)$ are calculated as follows, [28] (for more details see also Appendix 6):

$$
\begin{gathered}
W_{i}^{-}=\left|I_{i}^{-} \frac{\dot{\theta}_{i}^{b}+\dot{\theta}_{i}^{-}}{2}\right| \text { if } \dot{\theta}_{i}^{b} \dot{\theta}_{i}^{-} \geq 0 i=1,2,3,4,5 \\
W_{i}^{-}=\left|\frac{I_{i}^{-}}{2} \frac{\left(\dot{\theta}_{i}^{b}\right)^{2}+\left(\dot{\theta}_{i}^{-}\right)^{2}}{\dot{\theta}_{i}^{-}-\dot{\theta}_{i}^{b}}\right| \quad \text { if } \dot{\theta}_{i}^{b} \dot{\theta}_{i}^{-}<0 i=1,2,3,4,5 .
\end{gathered}
$$

Values $\dot{\theta}_{i}^{b}(i=1,2,3,4,5)$ are the inter-link angular velocities just before the first sub-phase. Values $\dot{\theta}_{i}^{-}(i=1,2,3,4,5)$ are the inter-link angular velocities just after the first sub-phase (before the second sub-phase). These velocities are calculated using expressions (36):

$$
\begin{gathered}
\dot{\theta}_{1}^{b}=\dot{q}_{2}^{b}-\dot{q}_{1}^{b}, \dot{\theta}_{2}^{b}=\dot{q}_{5}^{b}-\dot{q}_{2}^{b}, \dot{\theta}_{3}^{b}=\dot{q}_{5}^{b}-\dot{q}_{3}^{b}, \dot{\theta}_{4}^{b}=\dot{q}_{3}^{b}-\dot{q}_{4}^{b}, \dot{\theta}_{5}^{b}=\dot{q}_{1}^{b}, \\
\dot{\theta}_{1}^{-}=\dot{q}_{2}^{-}-\dot{q}_{1}^{-}, \dot{\theta}_{2}^{-}=\dot{q}_{5}^{-}-\dot{q}_{2}^{-}, \dot{\theta}_{3}^{-}=\dot{q}_{5}^{-}-\dot{q}_{3}^{-}, \dot{\theta}_{4}^{-}=\dot{q}_{3}^{-}-\dot{q}_{4}^{-}, \dot{\theta}_{5}^{-}=\dot{q}_{1}^{-} .
\end{gathered}
$$

Values $W_{i}^{+}(i=1,2,3,4$, and 6$)$ are calculated using analogous formulas:

$$
\begin{gathered}
W_{i}^{+}=\left|I_{i}^{+} \frac{\dot{\theta}_{i}^{+}+\dot{\theta}_{i}^{a}}{2}\right| \quad \text { if } \dot{\theta}_{i}^{+} \dot{\theta}_{i}^{a} \geq 0 \quad i=1,2,3,4, \text { and } 6, \\
W_{i}^{+}=\left|\frac{I_{i}^{+}}{2} \frac{\left(\dot{\theta}_{i}^{+}\right)^{2}+\left(\dot{\theta}_{i}^{a}\right)^{2}}{\dot{\theta}_{i}^{a}-\dot{\theta}_{i}^{+}}\right| \quad \text { if } \dot{\theta}_{i}^{+} \dot{\theta}_{i}^{a}<0 \quad i=1,2,3,4 \text {, and } 6 .
\end{gathered}
$$


Values $\dot{\theta}_{i}^{+}(i=1,2,3,4$, and 6$)$ are the inter-link angular velocities just after the second sub-phase. Values $\dot{\theta}_{i}^{a}(i=1,2,3,4$, and 6) are inter-link angular velocities just after the third sub-phase (at the start of the next swing phase). These velocities are calculated using expressions (39):

$$
\begin{gathered}
\dot{\theta}_{1}^{+}=\dot{q}_{2}^{+}-\dot{q}_{1}^{+}, \dot{\theta}_{2}^{+}=\dot{q}_{5}^{+}-\dot{q}_{2}^{+}, \dot{\theta}_{3}^{+}=\dot{q}_{5}^{+}-\dot{q}_{3}^{+}, \dot{\theta}_{4}^{+}=\dot{q}_{3}^{+}-\dot{q}_{4}^{+}, \dot{\theta}_{6}^{+}=\dot{q}_{4}^{+}, \\
\dot{\theta}_{1}^{a}=\dot{q}_{2}^{a}-\dot{q}_{1}^{a}, \dot{\theta}_{2}^{a}=\dot{q}_{5}^{a}-\dot{q}_{2}^{a}, \dot{\theta}_{3}^{a}=\dot{q}_{5}^{-}-\dot{q}_{3}^{a}, \dot{\theta}_{4}^{a}=\dot{q}_{3}^{+}-\dot{q}_{4}^{a}, \dot{\theta}_{6}^{a}=\dot{q}_{4}^{a} .
\end{gathered}
$$

Now let us consider the biped with exoskeleton in the double-support phase. In this phase, the torque developed by the human is applied in the leg 1 (hind leg), but the torque developed by the brake device is applied in the knee of leg 2 (front leg). To evaluate the energy consumption $W_{d s}$ of the impulsive torques applied at the first and third sub-phases instead of (32) we consider:

$$
\mathbf{W}_{d s}=\sum_{i=2-5} \int_{T^{-}}^{T}\left|\Gamma_{i}(t) \dot{\theta}_{i}(t)\right| \mathrm{d} t+\sum_{i=1-3,6} \int_{T}^{T^{+}}\left|\Gamma_{i}(t) \dot{\theta}_{i}(t)\right| \mathrm{d} t .
$$

We took into account in expression (40) the torques developed by the human only. Expression (40) can be written in the following form:

$$
\mathbf{W}_{d s}=\sum_{i=2-5} W_{i}^{-}+\sum_{i=1-3,6} W_{i}^{+}
$$

The sum

$$
\mathbf{W}=\mathbf{W}_{s s}+\mathbf{W}_{d s}
$$

is used to evaluate the energy consumption in both phases.

In simulation, with given time period $T$ and length $L$ of the step, we choose a unique solution of the system (21) - (29) by minimizing the quantity respectively (33) for the biped alone and (41) for the biped with the exoskeleton. We take into account the following constraints:

$$
I_{r_{1 y}}^{-} \geq 0, V_{2_{y}}^{-} \leq 0, I_{r_{2 y}} \geq 0, V_{1_{y}} \geq 0, I_{r_{2 y}}^{+} \geq 0
$$

Constraint $V_{2_{y}}^{-} \leq 0$ means that the vector of the linear velocity of the swing leg (leg 2) tip just after the first sub-phase is directed to the ground (downwards). Constraint $V_{1_{y}} \geq 0$ means that just after the passive impact the vector of the linear velocity of the hind leg (leg 1) tip has to be directed from the ground (upwards). For the biped alone we have also to take into account the four following inequalities:

$$
\dot{\theta}_{1}^{-} \geq 0, \dot{\theta}_{4}^{-} \geq 0, \dot{\theta}_{1}^{+} \geq 0, \dot{\theta}_{4}^{+} \geq 0
$$

Therefore, our minimization problem is the problem of parametric minimization with constraints. We used the SQP method (Sequential Quadratic Programming); see [50] and [51] with the fmincon function or fgoalattain function of Matlab $\mathbb{R}$ to solve this problem numerically. These algorithms based on the gradient of the objective with respect to the optimization variables allow to take into account linear and non-linear constraints. Numerical results are quasi similar with both $S Q P$ methods.

The minimal quantity (33) or (41) corresponding to this solution is considered in the ensuing section as the energy costs of impulsive torques in double-support phase for the biped walking with given data $L$ and $T$. After we calculate the sum (42) which is the energy consumption in both phases. 


\section{Simulation results}

In Subsection 5.1, we show the stick-diagrams of the walking gait for the biped alone and with exoskeleton. Also the graphs of the ground reaction in the single-support motion are presented. In Subsection 5.2, we compare the energy consumption of the biped with exoskeleton carrying a payload with the energy consumption of the biped alone carrying the same payload.

5.1 Stick figures and ground reaction

The stick figures of the walking gaits are shown for the biped alone in Fig 7 and for the biped with the exoskeleton in Fig 8 respectively. For both cases the step length and step duration of the walking gait are $L=0.50 \mathrm{~m}$ and $T=0.45 \mathrm{~s}$ respectively.

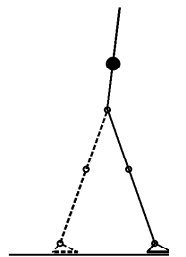

$t=0 \mathrm{~s}$

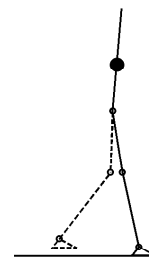

$t=0.09 \mathrm{~s}$

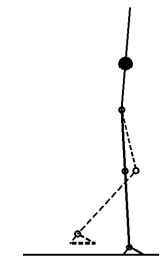

$t=0.18 s$

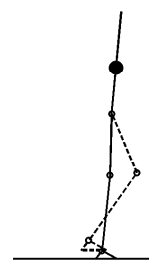

$t=0.27 \mathrm{~s}$

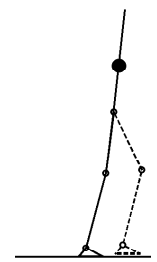

$t=0.36 \mathrm{~s}$

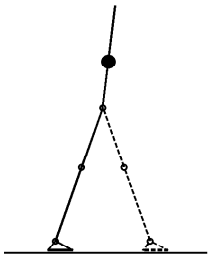

$t=0.45 \mathrm{~s}$

Fig. 7 Biped alone, walking ballistic gait as a sequence of stick figures.

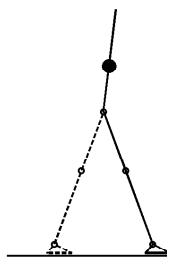

$t=0 s$

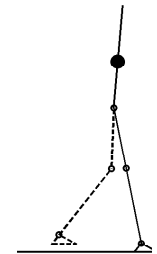

$t=0.09 \mathrm{~s}$

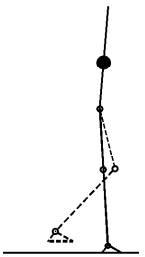

$t=0.18 s$

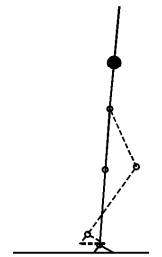

$t=0.27 \mathrm{~s}$

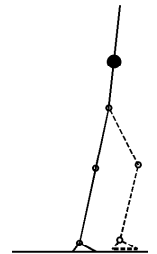

$t=0.36 \mathrm{~s}$

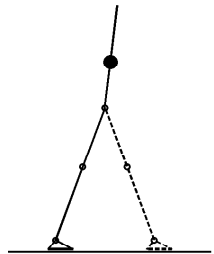

$t=0.45 s$

Fig. 8 Biped with exoskeleton, walking ballistic gait as a sequence of stick figures.

For the biped alone, we can observe a slight bending in the knee joint of the support leg at $t=0.27 \mathrm{~s}$ and $t=0.36 \mathrm{~s}$. It is never the case for the biped with the exoskeleton, that is consistent with condition (14). This condition means that the knee of the support leg is locked with the brake device and remains straight one during the single-support. The trunk of the biped with and without exoskeleton performs the oscillation with a "small" amplitude. 
The ground reaction components following $x$-axis and $y$-axis are shown for biped alone and with exoskeleton in Fig. $9(L=0.50 \mathrm{~m}, T=0.45 \mathrm{~s})$. At any time of the single-support motion the vertical component of the ground reaction for the biped with exoskeleton is positive and more than this component for the biped alone. The same thing takes place for the horizontal components: almost at any time of the singlesupport motion the horizontal component for the biped with exoskeleton is more, in absolute value, than this component for the biped alone. This is due to the exoskeleton, that its mass is $14 \mathrm{~kg}$. The difference in the shapes of the ground reaction is apparently due to the locking of the knee of the support leg of the biped with the exoskeleton. These observations are also validated by other combinations of parameters $L$ and $T$.

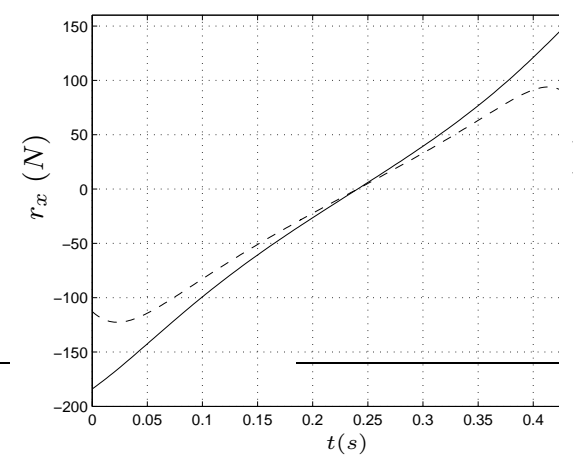

a)

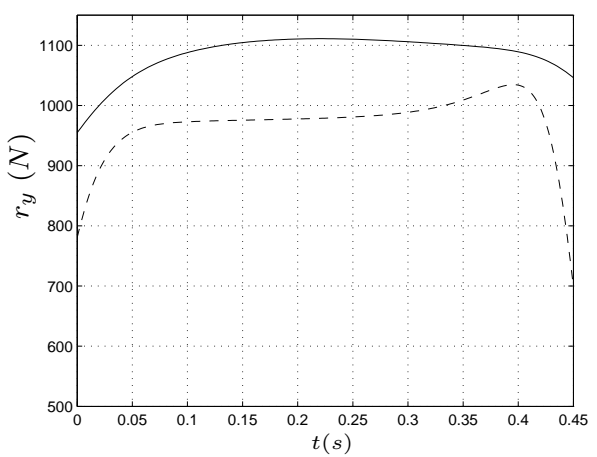

b)

Fig. 9 Ground reactions for biped alone (dashed lines) and for biped with exoskeleton (solid lines): a) horizontal components, b) vertical components.

We see from both stick diagrams (Figs 7 and 8) that the swing leg bends the knee forward and moves over the ground for $0<t<T$. From Figure 9 we see that the vertical component of the ground reaction in the support leg is positive (directed upwards), the sign of the horizontal component changes one time as in walking gait of human, see [52] where the anterior-posterior force has a similar behavior. Considering these figures we can conclude that the motion obtained in the simulation is natural and consequently admissible.

\subsection{Consumption of energy}

The consumption of energy is studied as a function of the step duration $T$ and of the step length $L$ for the biped alone and with the exoskeleton to transport the payload with mass $m_{c}=40 \mathrm{~kg}$. First, time $T$ varies from $0.4 \mathrm{~s}$ to $0.52 \mathrm{~s}$ while the length $L$ is fixed at $0.5 \mathrm{~m}$. Then $L$ varies from $0.40 \mathrm{~m}$ to $0.52 \mathrm{~m}$ while the time $T$ is fixed at $0.45 \mathrm{~s}$. 


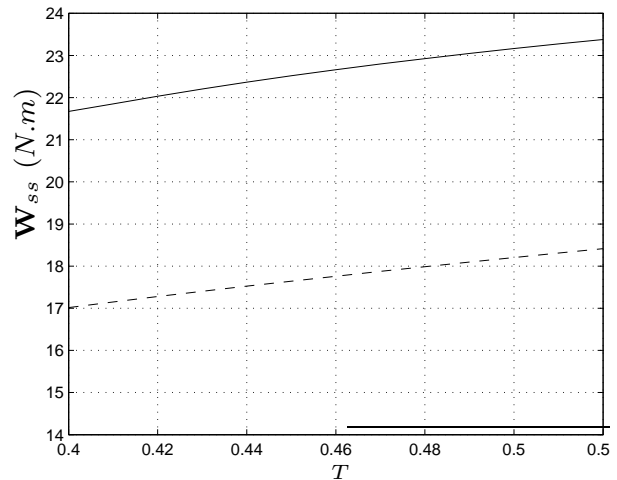

a)

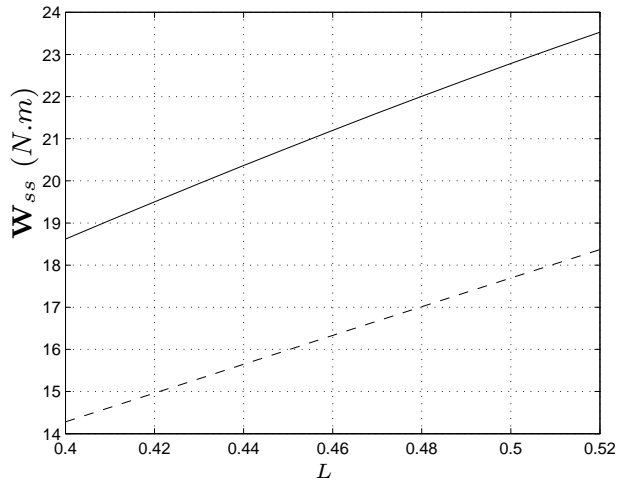

b)

Fig. 10 a) $W_{s s}$ as function of $T, L=0.5 \mathrm{~m}$. b) $W_{s s}$ as function of $L, T=0.45 \mathrm{~s}$. Dashed lines are used for biped alone, solid lines - for biped with exoskeleton.

Figure 10 shows that the energy consumption in the single-support motion is higher for the biped with exoskeleton than alone. It is logical from the physical point of view because the inertia moment with respect to the ankle of the support leg of the biped with exoskeleton is more (due to its mass $14 \mathrm{~kg}$ ) than of the biped alone.

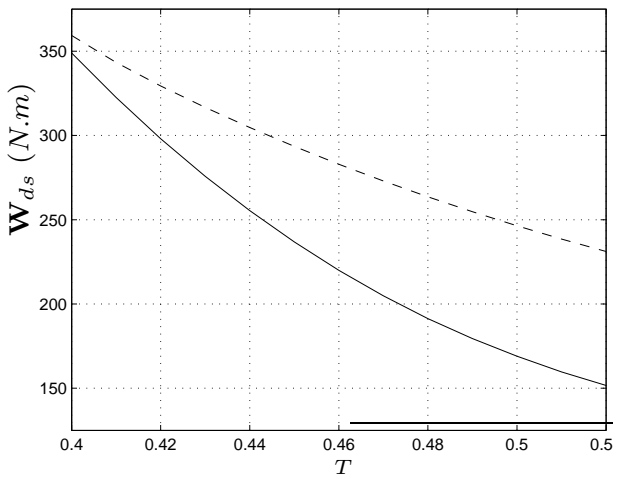

a)

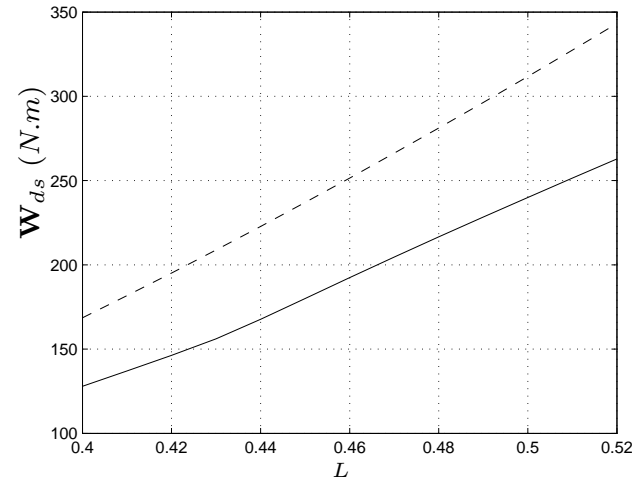

b)

Fig. 11 a) $W_{d s}$ as function of $T, L=0.5 \mathrm{~m}$. b) $W_{d s}$ as function of $L, T=0.45 \mathrm{~s}$. Dashed lines for biped alone, solid lines for biped with exoskeleton.

The energy consumption of the biped at the instantaneous double-support is much more than during the single-support motion. It is true for the biped alone and for the biped equipped with exoskeleton, see Figs 10, 11. This result corresponds to the opinion of some researchers that most of the efforts during human walking take place in the double-support (see Mochon and McMahon [31]). 


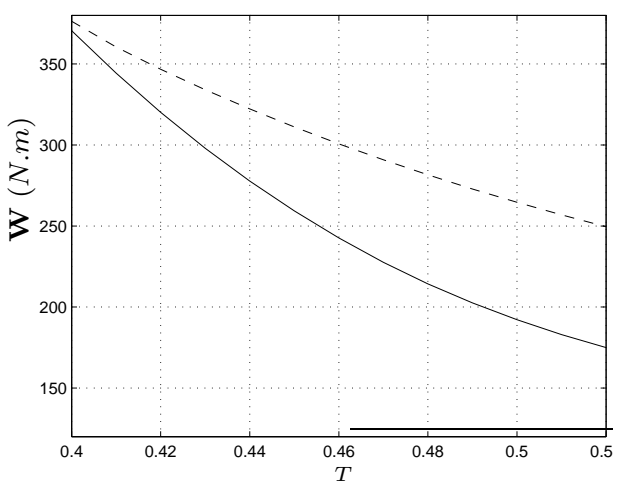

a)

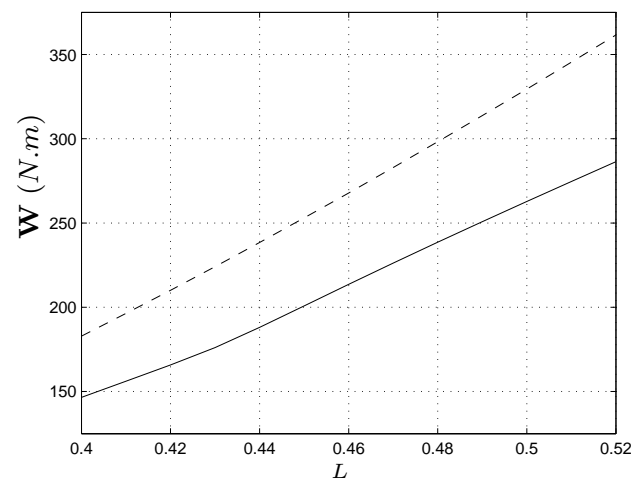

b)

Fig. 12 a) $W$ as function of $T, L=0.5 \mathrm{~m}$. b) $W$ as function of $L, T=0.45 \mathrm{~s}$. Dashed lines for biped alone, solid lines for biped with exoskeleton.

Figures 12 show the energy value which is equal to the sum (42) of the energies provided by the biped (human) during the single-support motion and in the instantaneous double-support phase. The biped with the exoskeleton is more efficient from the point of view of the energy consumption than the biped alone. The same property is observed when the duration $T$ of the step increases (with fixed step length $L$ ) or the step length $L$ increases (with fixed step time $T$ ). This means that the exoskeleton is useful in the corresponding ranges of the step lengths and durations. The economy of the energy is approximately $28 \%$, if the length of the step and its duration are respectively equal to $0.5 \mathrm{~m}$ and $0.5 \mathrm{~s}$.

\section{Conclusion}

Planar anthropomorphic bipedal mechanism with massless feet is considered as mechanical model of a human walking. An exoskeleton to assist human is also considered as a bipedal mechanism (massive, but with massless feet) with the same shape and degrees of freedom as mechanical model of the human alone. The links of the exoskeleton are strongly strapped to the corresponding links of the biped. We study passive exoskeleton without any sources of energy and actuators; the knee of the support leg of our exoskeleton (and as consequence of the human) is locked using mechanical brake device, but the knee of the swing leg is unlocked. For the biped (with or without exoskeleton) the progression of the $C O P$ of the support leg is prescribed as a polynomial function of time. An actuation torque is applied in the ankle-joint of the support leg.

It is theoretically shown the efficiency of mentioned above passive exoskeleton for human carrying a load. The human equipped with the exoskeleton spends less energy than human alone, despite the fact that the mass of the exoskeleton is taken into account. In our study, this mass is near $11 \%$ of mass of human with load and exoskeleton. The study is done in the framework of the ballistic walking problem. Mathematically this problem is reduced to the solving the boundary value problem for the motion equations of the single-support and to design the impulsive torques for the instantaneous double-support. Many theoretical problems are still open, and an investigation in 
$3 \mathrm{D}$ is necessary. However, the concept of the passive exoskeleton seems very interesting.

Acknowledgement: This work is supported by Ministry of Education and Science of Russian Federation, Project No. 7.524.11.4012, and by Région des Pays de la Loire, Project $L M A$ and Gérontopôle Autonomie Longévité des Pays de la Loire. 
Appendix: Energy consumption at the instantaneous double-support phase

If the impulsive torques described by Dirac delta-functions are applied at the interlink joints of the biped, then each interlink angular velocity undergoes a discontinuous change.

For a sake of clarity and without loss of generality we consider only one actuated joint of the biped. The joint variable is defined with $\theta$. An impulsive torque $\Gamma$ applied at this joint is defined to be as follows:

$$
\Gamma(t)=I \delta(t-T) .
$$

Expression (45) describes the Dirac delta-function; the magnitude $I$ describes the intensity of the torque $\Gamma$ such that:

$$
\int_{T^{-}}^{T^{+}} I \delta(t-T) \mathrm{d} t=I .
$$

We want to evaluate the energy expended during the operation of the impulsive torque (45). The chosen energy criterion is as follows:

$$
W=\int_{T^{-}}^{T+}|I \delta(t-T)(t) \dot{\theta}(t)| \mathrm{d} t
$$

Now instead of delta-function (45) let us consider the following distributed in time piecewise constant function:

$$
\Gamma_{\Delta}(t)=\left\{\begin{array}{c}
\frac{I}{2 \Delta} \text { if } t \in[T-\Delta, T+\Delta] \\
0 \text { if } t \notin[T-\Delta, T+\Delta]
\end{array}\right.
$$

Here $\Delta=$ const $>0$; function (47) is shown in Fig. 13. If $\Delta \rightarrow 0$, then function $\Gamma_{\Delta}(t)$ "tends" to Dirac delta-function (45).

Let us assume that in each interlink joint the distributed in time torque (similar to (47)) is applied. And let value $\Delta$ be the same for each joint.

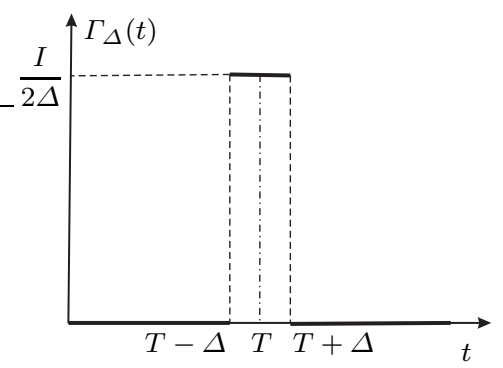

Fig. 13 Function $\Gamma_{\Delta}(t)$. 
The joint velocity $\dot{\theta}$ undergoes discontinuity at the instant $T$ (as in each joint), when the impulsive torques (similar to torque (45)) are applied in the joints of our biped. Let equality $\dot{\theta}=\dot{\theta}^{-}$be just before the applying of the impulsive torques and equality $\dot{\theta}=\dot{\theta}^{+}$be just after the applying of these impulsive torques.

If interval $[T-\Delta, T+\Delta]$ is "small", then velocity $\dot{\theta}(t)$ can be distributed in this interval by the following way (function $O(\Delta)$ is magnitude of the first order with respect to magnitude $\Delta$ )

$$
\dot{\theta}_{\Delta}(t)=\dot{\theta}^{-}+\frac{\dot{\theta}^{+}-\dot{\theta}^{-}}{2 \Delta}(t-T+\Delta)+O(\Delta)
$$

If $\Delta \rightarrow 0$, then according to expression (48) $O(\Delta) \rightarrow 0$ and $\dot{\theta}_{\Delta}(T-\Delta) \rightarrow \dot{\theta}^{-}, \dot{\theta}(T+$ $\Delta) \rightarrow \dot{\theta}^{+}$.

Now let us consider instead of (46) the following integral using expressions (47) and (48):

$$
W_{\Delta}=\int_{T-\Delta}^{T+\Delta}\left|\Gamma_{\Delta}(t) \dot{\theta}_{\Delta}(t)\right| \mathrm{d} t=\left|\frac{I}{2 \Delta}\right| \int_{T-\Delta}^{T+\Delta}\left|\dot{\theta}^{-}+\frac{\dot{\theta}^{+}-\dot{\theta}^{-}}{2 \Delta}(t-T+\Delta)+O(\Delta)\right| \mathrm{d} t .
$$

To calculate integral (49) we consider two cases. First case is as follows:

$$
\dot{\theta}^{-} \dot{\theta} \geqslant 0 \text { and } \dot{\theta}^{-} \neq 0 \text { or } \dot{\theta}^{+} \neq 0
$$

It is possible to show that under condition (50)

$$
\begin{aligned}
\lim _{\Delta \rightarrow 0} W_{\Delta} & =\lim _{\Delta \rightarrow 0}\left|\frac{I}{2 \Delta}\right| \int_{T-\Delta}^{T+\Delta}\left|\dot{\theta}^{-}+\frac{\dot{\theta}^{+}-\dot{\theta}^{-}}{2 \Delta}(t-T+\Delta)+O(\Delta)\right| \mathrm{d} t \\
& =\left|\frac{I}{2 \Delta}\right| \int_{T-\Delta}^{T+\Delta}\left|\dot{\theta}^{-}+\frac{\dot{\theta}^{+}-\dot{\theta}^{-}}{2 \Delta}(t-T+\Delta)\right| \mathrm{d} t=\left|I \frac{\dot{\theta}^{+}+\dot{\theta}^{-}}{2}\right| .
\end{aligned}
$$

The expression of the last integral in (51) contains value $\Delta$. However, the result of its calculation does not depend on this value and this result looks like (34) or (37) for the joint $i$.

Let us consider now the second case, when

$$
\dot{\theta}^{-} \dot{\theta}^{+}<0
$$

Function (48) becomes zero at the instant

$$
t_{0}=T-\Delta+\frac{2 \dot{\theta}^{-}}{\dot{\theta}^{-}-\dot{\theta}^{+}} \Delta+O\left(\Delta^{2}\right) .
$$


Function (48) has different signs in intervals $\left[T-\Delta, t_{0}\right)$ and $\left(t_{0}, T+\Delta\right]$. Therefore, integral (49) can be written as follows:

$$
\begin{aligned}
W_{\Delta}= & \left|\frac{I}{2 \Delta}\right| \mid \int_{T-\Delta}^{t_{0}}\left[\dot{\theta}^{-}+\frac{\dot{\theta}^{+}-\dot{\theta}^{-}}{2 \Delta}(t-T+\Delta)+O(\Delta)\right] \mathrm{d} t \\
& -\int_{t_{0}}^{T+\Delta}\left[\dot{\theta}^{-}+\frac{\dot{\theta}^{+}-\dot{\theta}^{-}}{2 \Delta}(t-T+\Delta)+O(\Delta)\right] \mathrm{d} t \mid .
\end{aligned}
$$

Straightforward calculations show that

$$
\begin{aligned}
\lim _{\Delta \rightarrow 0} W_{\Delta}= & \left|\frac{I}{2 \Delta}\right| \mid \int_{T-\Delta}^{t_{0}^{*}}\left[\dot{\theta}^{-}+\frac{\dot{\theta}^{+}-\dot{\theta}^{-}}{2 \Delta}(t-T+\Delta)\right] \mathrm{d} t \\
& -\int_{t_{0}^{*}}^{T+\Delta}\left[\dot{\theta}^{-}+\frac{\dot{\theta}^{+}-\dot{\theta}^{-}}{2 \Delta}(t-T+\Delta)\right] \mathrm{d} t|=| \frac{I}{2} \frac{\left(\dot{\theta}^{+}\right)^{2}+\left(\dot{\theta}^{-}\right)^{2}}{\dot{\theta}^{+}-\dot{\theta}^{-}} \mid
\end{aligned}
$$

Here $t_{0}^{*}=T-\Delta+\frac{2 \dot{\theta}^{-}}{\dot{\theta}^{-}-\dot{\theta}^{+}} \Delta$. The expression of each integral in (55) contains value $\Delta$, but the calculations show that the difference between these two integrals does not depend on this value. The result of these calculations looks like (35) and (38) for the joint $i$.

\section{References}

1. A. Dollar and H. Herr, "Lower extremity exoskeletons and active orthoses: Challenges and state-of-the-art," IEEE Trans. on Robotics, vol. 24, no. 1, pp. 144-158, 2008.

2. T. Hayashi, T. Sakurai, and K. Eguchi, "Development of single leg version of hal for hemiplegia," in Proc. Int. Conf. of the IEEE Engineering in Medicine and Biology Society, Minneapolis, USA, 2009, pp. 5038-5043, DOI: 10.1109/IEMBS.2009.5 333698.

3. S. Viteckova, P. Kutilek, and M. Jirina, "Wearable lower limb robotics: a review," Biocybernetics and Biomedical Engineering, vol. 33, no. 2, pp. 96-105, 2013.

4. M. Talaty, A. Esquenazi, and J. E. Briceno, "Differentiating ability in users of the rewalktm powered exoskeleton: An analysis of walking kinematics," in Proc. IEEE Int. Conf. on Rehabilitation Robotics (ICORR), Seattle, USA, 2013, pp. 1-5, DOI: 10.1109/ICORR.2013.6 650469.

5. B. S. Rupala, A. Singla, and G. S. Virk, "Lower limb exoskeletons: A brief review," in Proc. Int. Conf. on Mechanical Engineering \& Technology COMET, Varanasi, Utter Pradesh, 2016, pp. 18-24.

6. R. J. Farris, H. A. Quintero, S. A. Murray, K. H. Ha, C. Hartigan, and M. Goldfarb, "A preliminary assessment of legged mobility provided by a lower limb exoskeleton for persons with paraplegia," IEEE Trans. on Neural, Systems and Rehabilitation Engineering, vol. 22, no. 3, pp. 482-490, 2014.

7. Y. Aoustin, "Walking gait of a biped with a wearable walking assist device," Int. J. of Humanoid Robotics, vol. 12, no. 2, pp. 1550018-1-11550018-20, DOI: 10.1142/S0 219843615500 188, 2015.

8. S. J. Zaroodny, "Bumpusher: A powered aid to locomotion," U. S. Army Ballistic res. Lab., Aberdeen Proving Ground, MD, Tech. Note 1524, 1963. 
9. M. Vukobratovic, D. Hristic, and Z. Stojiljkovic, "Development of active anthropomorphic exoskeletons," Med. Biol. Eng., vol. 12, no. 1, pp. 66-80, 1974.

10. J. Main, "Exoskeletons for human performance augmentation," in DARPA Project, 3701 North Fairfax Drive, Arlington, 2005.

11. A. Zoss, H. Kazerooni, and H. Chu, "On the mechanical design of the berkeley lower extremity exoskeleton (bleex)," in Proc. IEEE/RSJ Int. Conf. on Intelligent Robots and Systems, Alberta, Canada, 2005, pp. 3465-3472.

12. H. Kazerooni and R. Steger, "The berkeley lower extremity exoskeleton," Trans. ASME J. Dynamic, Systems, Measurement, and Control, vol. 128, no. 1, pp. 14-25, 2006.

13. S. Marcheschi, F. Salsedo, M.Fontana, and M. Bergamasco, "Body extended: whole body exoskeleton for human power augmentation," in Proc. IEEE Int. Conf. on Robotics and Automation, Shanghai China, 2011, pp. 611-616.

14. T. Yana, M. Cempini, C. M. Oddo, and N. Vitiello, "Review of assistive strategies in powered lower-limb orthoses and exoskeletons," Robotics and Autonomous Systems, vol. 64, pp. 120-136, 2015.

15. H. Herr, "Exoskeletons and orthoses: classification, design, design challenges and future directions," J. of NeuroEngineering and Rehabilitation, vol. 6:21, pp. 1-9, doi:10.1186/17430003-6-21, 2009.

16. K. A. Strausser and H. Kazerooni, "The development and testing of a human machine interface for a mobile medical exoskeleton," in IEEE. Int. Conf. on Intelligent Robots and Systems, San Francisco, USA, 2011, pp. 4911-4916.

17. A. J. van den Bogert, "Exotendons for assistance of human locomotion," Biomedical Engineering Online, pp. 2-17, 2003.

18. C. J. Walsh, K. Pasch, and H. Herr, "An autonomous, under actuated exoskeleton for load-carrying augmentation," in Proc. IEEE/RSJ Int. Conf. on Intelligent Robots and Systems, Beijing China, 2006, pp. 1410-1415.

19. S. K. Agrawal, S. K. Banala, A. Fattah, J. P. Scholz, V. Krishnamoorthy, and W.-L. Hsu, "A gravity balancing passive exoskeleton for the human leg," in Robotics: Science and Systems, 2006.

20. S. K. Agrawal, S. K. Banala, A. Fattah, V. Sangwan, V. Krishnamoorthy, J. P. Scholz, and W. L. Hsu, "Assessment of motion of a swing leg and gait rehabilitation with a gravity balancing exoskeleton," IEEE Trans. on Neural Systems and Rehabilitation Enginneering, vol. 15 , no. 3 , pp. 410-420, 2007.

21. B. Dariush, "Analysis and simulation of an exoskeleton controller that accommodates static and reactive loads," in Proc. IEEE Conf. on Robotics and Automation, Barcelona, Spain, 2005, pp. 2350-2355.

22. W. van Dijk, H. van der Kooij, and E. Heckman, "A passive exoskeleton with artificial tendons," in IEEE. Int. Conf. on Rehabilitation Robotics, Rehab Week Zurich, ETH Zurich Science City, Switzerland, June 29-July 1, 2011.

23. R. Xi, Z. Zhu, F. Du, M. Yang, X. Wang, and Q. Wu, "Deign concept of the quasi-passive energy-efficient power-assisted lower-limb exoskeleton based on the theory of passive dynamic walking," in Proc. Int. Conf. of the 23rd IEEE on Mechatronics and Machine Vision in Practice (M2VIP), Nanjing, China, 2016, pp. 1-5.

24. A. Collo, V. Bonnet, and G. Venturei, "A quasi-passive lower limb exoskeleton for partial body weight support," in Proc. Int. Conf. of the 6th IEEE RAS/EMBS Engineering on Biomedical Robotics and Biomechatronics (BioRob), UTown, Singapore, 2016, pp. 643648.

25. Y. Aoustin and A. M. Formalskii, Strategy to Lock the Knee of Exoskeleton Stance Leg: Study in the Framework of Ballistic Walking Model. New Trends in Medical and Service Robots: Human Walking, Eds. P. Wenger, C. Chevallereau, Doina Pisla, Hannes Bleuler, and A. Rodic. 275p, 2016.

26. R. Dumas, L. Chèze, and J. P. Verriest, "Adjustments to mcconville et al. and young et al. body segment parameters," J. of Biomechanics, vol. 40, no. 3, pp. 543-553, 2007.

27. A. M. Formal'skii, "Motion of anthropomorphic biped under impulsive control," in Proc. of Institute of Mechanics, Moscow State Lomonosov University: "Some Questions of Robot's Mechanics and Biomechnics", 1978, (In Russian), pp. 17-34.

28. A. M. Formalskii, Locomotion of Anthropomorphic Mechanisms. [In Russian], Nauka, Moscow, Russia, 1982.

29. A. Formal'sky, Ballistic Locomotion of a Biped. Design and Control of Two Biped Machines. Human and Machine Locomotion, Eds. A. Morecki and K. Waldron. SpringerVerlag, 1997. 
30. A. M. Formal'skii, "Ballistic walking design via impulsive control," ASCE, Journal of Aerospace Engineering, vol. 23, no. 2, pp. 129-138, 2010.

31. S. Mochon and T. McMahon, "Ballistic walking: An improved model," Mathematical Biosciences, vol. 52, pp. 241-260, 1981.

32. T. McGeer, "Passive dynamic walking," Int. J. of Robotics Research, vol. 9, no. 2, pp. 62-82, 1990.

33. Y. Aoustin and A. M. Formalskii, "3D walking biped: optimal swing of the arms," Multibody System Dynamics, vol. 32, no. 1, DOI 10.1007/s11044-013-9378-3, pp. 55-66, 2014.

34. M. Wisse, Essentials of dynamic walking, Analysis and design of two legged robots. Phd Thesis, ISBN 90-77595-82-1, 2004.

35. S. Collins, S. Ruina, R. Tedrake, and M. Wisse, "Efficient bipedal robots based on passivedynamic walkers," Science Magazine, no. 19, pp. 1082-1085, 2005.

36. J. B. Geursen, D. Altena, and C. H. Massen, "A model of the standing man for the description of his dynamic behaviour," Agressologie, vol. 17, no. 12, pp. 63-69, 1976.

37. W. O. Fenn, "Work against gravity and work due to velocity changes in running," Americain Journal of Physiology, vol. 93, pp. 433-462, 1930.

38. G. A. Cavagna, H. Thys, and A. Zamboni, "The sources of external work in level walking and running," The Journal of Physiology, vol. 261, no. 3, pp. 639-657, 1976.

39. J. L. Patton, Y. C. Pai, and W. A. Lee, "Evaluation of a model that determines the stability limits of dynamic balance," Gait \& Posture, vol. 9, no. 1, pp. 38-49, 1999.

40. Y. Ikeuchi, J. Ashihara, Y. Hiki, H. Kudoh, and T. Noda, "Walking assist device with bodyweight support system," in Proc. IEEE/RSJ Int. Conf. on Intelligent Robots and Systems, St Louis, USA, 2010, pp. 4073-4079.

41. P. Appell, Dynamique des Systèmes - Mécanique Analytique. Paris, P. Gauthiers-Villars, 1931.

42. M. Vukobratovic and B. Borovac, "Zero-moment point-thirty five years of its life," Int. J. of Humanoid Robotics, vol. 1, no. 1, pp. 157-173, 2004.

43. S. Devie and S.Sakka, Effects of the rolling mechanism of the stance foot on the generalized inverted pendulum definition. New Trends in Medical and Service Robots: Human Walking, Eds. P. Wenger, C. Chevallereau, Doina Pisla, Hannes Bleuler, and A. Rodic. $275 p, 2016$.

44. N. J. Rosenblatt and M. D. Grabiner, "Measures of frontal plane stability during treadmill and overground walking," Gait Posture, vol. 31, no. 3, pp. 380-384, 2010.

45. V. Lugade and K. Kaufman, "Center of pressure trajectory during gait: A comparaison of four foot positions," Gait Posture, vol. 40, no. 1, pp. 252-254, 2014.

46. J. M. Font-Llagunes, A. M. Barjau, R. Pàmies, and V. J. Kövecses, "Dynamic analysis of impact in swing-through crutch gait using impulsive and continuous contact models," Multibody System Dynamics, vol. 28, no. 3, pp. 257-282, 2012.

47. Y. Hurmuzlu and T.-H. Chang, "Rigid body collisions of a special class of planar kinematic chains," IEEE Transactions on systems, man and cybernetics, vol. 22, no. 5, pp. 964-971, 1992.

48. A. Formal'skii, C. Chevallereau, and B. Perrin, "On ballistic walking locomotion of a quadruped," Int. J. of Robotics Research, vol. 19, no. 8, pp. 743-761, 2000.

49. V. V. Beletskii, Biped walking. [In Russian], Nauka, Moscow, Russia, 1984.

50. P. Gill, W. Murray, and M. Wright, Practical optimization. London: Academic Press, 1981.

51. M. Powell, Variable metric methods for constrained optimization, ser. Lecture Notes in Mathematics. Springer Berlin / Heidelberg, 1977, pp. 62-72.

52. Y. Jung, M. Jung, K. Lee, and S. Koo, "Ground reaction force estimation using an insoletype pressure mat and joint kinematics during walking," Journal of Biomechanics, vol. 47, pp. 2693-2699, 2014.

53. L. Schwartz, Méthodes mathématiques pour les sciences physiques. Editions Hermann, Enseignement des sciences, 1965.

54. P. ORHANT, "Contribution a la manipulation fine et étude de la phase d'impact," Ph.D. dissertation, INPG Grenoble France, 1994, thèse de doctorat dirigée par CANUDAS DE WIT, C. Sciences appliquées Grenoble INPG 1994. [Online]. Available: http://www.theses.fr/1994INPG0171 